Pacific

Journal of

Mathematics

\title{
COMPLETELY CONTRACTIVE PROJECTIONS ON OPERATOR ALGEBRAS
}

David P. Blecher AND Matthew Neal 


\title{
COMPLETELY CONTRACTIVE PROJECTIONS ON OPERATOR ALGEBRAS
}

\author{
David P. BLEChER AND MATthew NeAL
}

\begin{abstract}
The main goal of this paper is to find operator algebra variants of certain deep results of Størmer, Friedman and Russo, Choi and Effros, Effros and Størmer, Robertson and Youngson, Youngson, and others, concerning projections on $C^{*}$-algebras and their ranges. In particular, we investigate the "bicontractive projection problem" and related questions in the category of operator algebras. To do this, we will add the ingredient of "real positivity" from recent papers of the first author with Read.
\end{abstract}

\section{Introduction}

In previous papers (listed in the bibliography) both authors separately studied projections (that is, idempotent linear maps) and conditional expectations on unital operator algebras (that is, closed algebras of operators on a Hilbert space that contain the identity operator) and other classes of Banach spaces. Results were proved such as Corollary 4.2.9 in [Blecher and Le Merdy 2004]: A completely contractive projection $P$ on such an algebra $A$ which is unital (that is, $P(1)=1)$ and whose range is a subalgebra is a conditional expectation (that is, $P(P(a) b P(c))=P(a) P(b) P(c)$ for $a, b, c \in A)$. This is an analogue of the matching theorem due to Tomiyama for $C^{*}$-algebras.

The main goal of our paper is to find variants, valid for operator algebras which are unital or which have an approximate identity, of certain deeper results in the $C^{*}$-algebra case found in [Størmer 1982; Friedman and Russo 1984; 1985; Effros and Størmer 1979; Robertson and Youngson 1982], and elsewhere, concerning projections and their ranges. In particular we wish to investigate the "bicontractive projection problem" and related problems (such as the "symmetric projection problem" and the "contractive projection problem") in the category of operator algebras. To do this, we will add the ingredient of real positivity from recent papers

Blecher was partially supported by a grant from the National Science Foundation. Neal was supported by Denison University.

MSC2010: primary 46L05, 46L07, 47L07, 47L30; secondary 46H10, 46B40, 46L30.

Keywords: operator algebra, completely contractive map, projection, conditional expectation,

bicontractive projection, real positive, noncommutative Banach-Stone theorem. 
of Blecher and Read [2011; 2013; 2014]; see also [Blecher and Neal 2012a; 2012b; Bearden et al. 2014; Blecher and Ozawa 2015; Blecher 2015]. A key idea in those papers is that real positivity is often the right replacement in general algebras for positivity in $C^{*}$-algebras. This will be our guiding principle here too.

We now discuss the structure of our paper. In Section 2 we discuss completely contractive projections on operator algebras. A well-known and lovely result of Choi and Effros [1977, Theorem 3.1] (or rather, its proof) shows that the range of a completely positive projection $P: B \rightarrow B$ on a $C^{*}$-algebra $B$, is again a $C^{*}$-algebra with product $P(x y)$. A quite deep theorem of Friedman and Russo [1985], or a simpler variant of it by Youngson [1983], shows that something similar is true if $P$ is simply contractive, or if $B$ is replaced by a ternary ring of operators. The analogous result for unital completely contractive projections on unital operator algebras is true too, and is implicit in the proof of the result quoted in the first paragraph. However, there seems to be no analogous result for (nonunital) completely contractive projections on nonunital operator algebras without adding extra hypotheses on $P$. The "guiding principle" in the previous paragraph suggests to add the condition that $P$ is also "real completely positive" (we define this below). Then the question does make good sense, and we are able to prove the desired result. Thus the range of a real completely positive completely contractive projection $P: A \rightarrow A$ on an operator algebra with approximate identity is again an operator algebra with product $P(x y)$. We also have a converse and several complements to this result in Section 2, as well as some other facts about completely contractive projections, such as how one is often able to reduce the problem to algebras which have an identity. We also show that for algebras with no kind of approximate identity, there is a biggest "nice part" on which completely contractive projections (and the other classes of projections discussed below) work well.

In Sections 3, 4, and 5, we turn from the "contractive projection problem" to the "bicontractive projection problem" and related questions. A projection $P$ is bicontractive if both $P$ and $I-P$ are contractive. By the bicontractive projection problem for a Banach space $X$, one usually means the characterization of all bicontractive projections $P: X \rightarrow X$, or the characterization of the ranges of the bicontractive projections (or both). On a unital $C^{*}$-algebra $B$ it is known, by work of some of the authors mentioned above, that the unital bicontractive projections are precisely $\frac{1}{2}(I+\theta)$, for a period-2 $*$-automorphism $\theta: B \rightarrow B$. The possibly nonunital bicontractive projections $P$ on $B$ are of a similar form, and indeed if $P$ is also positive then $q=P(1)$ is a central projection in the multiplier algebra $M(B)$ with respect to which $P$ decomposes into a direct sum of 0 and a projection of the above form $\frac{1}{2}(I+\theta)$, for a period- $2 *$-automorphism $\theta$ of $q B$. (See Theorem 3.2 for the idea of the proof of this.) Conversely, note that a map $P$ of the latter form is automatically completely bicontractive (that is, is bicontractive at each matrix 
level), indeed is completely symmetric (that is, $I-2 P$ is completely contractive), and the range of $P$ is a $C^{*}$-subalgebra, and $P$ is a conditional expectation.

One may ask: what from the last paragraph is true for general operator algebras? Again the guiding principle referred to earlier leads us to use in place of the positivity in that result, the real positivity in the sense of [Blecher and Read 2013; 2014]. The next thing to note is that now "completely bicontractive" is no longer the same as "completely symmetric" for projections. The "completely symmetric" case works beautifully, and the solution to our "symmetric projection problem" is presented in Theorem 3.7. This result is one somewhat satisfactory generalization (we shall see others later) to operator algebras of the $C^{*}$-algebraic theorem in the last paragraph. For the more general class of completely bicontractive projections, as seems to be often the case in generalizing $C^{*}$-algebraic theory to more general algebras, a first look is disappointing. Indeed most of the last paragraph no longer works in general. One does not always get an associated completely isometric automorphism $\theta$ with $P=\frac{1}{2}(I+\theta)$, and $q=P(1)$ need not be a central projection. Indeed we have solved here and elsewhere (see, e.g., [Blecher and Labuschagne 2003; Blecher 2004; Blecher and Magajna 2005a, p. 92-93; 2005b]) many of the obvious questions about contractive projections, completely contractive projections, and conditional expectations, on operator algebras. Unfortunately many of the answers are counterexamples. However, as also seems to be often the case, a closer look at examples reveals an interesting question. Namely, given a real completely positive projection $P: A \rightarrow A$ which is completely bicontractive, when is the range of $P$ an (approximately unital) subalgebra of $A$, so that $P$ is a conditional expectation? For operator algebras we consider this to be the correct version of the bicontractive projection problem. In Sections 3 and 4 we elucidate this question. We remark that in [Blecher et al. $\geq 2016$ ] we study the "Jordan algebra" variants of many of the results in Sections 2 and 3 of the present paper.

In Section 4 we discuss the completely bicontractive projection problem, construct some interesting examples, and give some reasonable conditions under which $P(A)$ is a subalgebra and $P$ is a conditional expectation. In particular we solve in full generality our version of the bicontractive projection problem for uniform algebras (that is, closed subalgebras of $C(K)$ ), and indeed for any algebra satisfying a condition related to semisimplicity. Theorem 4.9 is one of the main results of the paper, giving a very general condition for $P(A)$ being a subalgebra in terms of certain support projections. In fact, at the time of writing, for all we know the condition in Theorem 4.9 is necessary and sufficient; at least we have no examples to the contrary. In Section 5 we discuss another condition that completely bicontractive projections may satisfy, and examine some consequences of this. In Section 6 we discuss Jordan homomorphisms on operator algebras, and among other things, solve two natural "completely isometric problems", for Jordan subalgebras of operator 
algebras and for operator algebras, related to the noncommutative Banach-Stone theorem.

We now turn to precise definitions and notation. Any unexplained terms below can probably be found in [Blecher and Le Merdy 2004; Paulsen 2002; Blecher and Read 2011; 2013] or any of the other books on operator spaces. All vector spaces are over the complex field $\mathbb{C}$. The letters $K, H$ denote Hilbert spaces. If $X$ is an operator space we often write $X_{+}$for the elements in $X$ which are positive (i.e., at least 0 ) in the usual $C^{*}$-algebraic sense. We write $\operatorname{Ball}(X)$ for the set $\{x \in X:\|x\| \leq 1\}$. By an operator algebra we mean a not necessarily selfadjoint closed subalgebra of $B(H)$, the bounded operators on a Hilbert space $H$. We write $C^{*}(A)$ for the $C^{*}$-algebra generated by $A$, that is, the smallest $C^{*}$-subalgebra containing $A$. A unital operator space is a subspace $X$ of $B(H)$ or a unital $C^{*}$-algebra containing the identity (operator). We often write this identity as $1_{X}$. A map $T: X \rightarrow Y$ is unital if $T\left(1_{X}\right)=1_{Y}$. We say that an algebra is approximately unital if it has a contractive approximate identity (cai). For us a projection in an operator algebra $A$ is always an orthogonal projection lying in $A$, whereas a projection on $A$ is a linear idempotent map $P: A \rightarrow A$. If $A$ is a nonunital operator algebra represented (completely) isometrically on a Hilbert space $H$ then one may identify the unitization $A^{1}$ with $A+\mathbb{C} I_{H}$. The second dual $A^{* *}$ is also an operator algebra with its (unique) Arens product; this is also the product inherited from the von Neumann algebra $B^{* *}$ if $A$ is a subalgebra of a $C^{*}$-algebra $B$. Note that $A$ has a cai if and only if $A^{* *}$ has an identity $1_{A^{* *}}$ of norm-1, and then $A^{1}$ is sometimes identified with $A+\mathbb{C} 1_{A^{* *}}$. The multiplier algebra $M(A)$ of such $A$ may be taken to be the idealizer of $A$ in $A^{* *}$, that is, $\left\{\eta \in A^{* *}: \eta A+A \eta \subset A\right\}$.

If $A$ is an approximately unital operator algebra or unital operator space then $I(A)$ denotes the injective envelope, an injective unital $C^{*}$-algebra containing $A$. It contains $A$ as a subalgebra if $A$ is approximately unital [Blecher and Le Merdy 2004, Corollary 4.2.8]. For us the most important properties of $I(A)$ are: first, that it is injective in the category of operator spaces, so that any completely contractive map from a subspace of an operator space $Y$ into $I(A)$ extends to a complete contraction from $Y$ to $I(A)$. Second, $I(A)$ is rigid, so that the identity map on $I(A)$ is the only complete contraction $I(A) \rightarrow I(A)$ extending the identity map on $A$. The $C^{*}$-envelope $C_{\mathrm{e}}^{*}(A)$ is the $C^{*}$-subalgebra of $I(A)$ generated by $A$. If $A$ is unital it has the property that given any unital complete isometry $T: A \rightarrow B(K)$, there exists a unique $*$-homomorphism $\pi: C^{*}(A) \rightarrow C_{\mathrm{e}}^{*}(A)$ with $\pi \circ T$ equal to the inclusion map of $A$ in $C_{\mathrm{e}}^{*}(A)$.

We recall that a contractive completely positive map on a $C^{*}$-algebra is completely contractive. A unital linear map between operator systems is positive and $*$-linear if it is contractive; and it is completely positive if and only if it is completely contractive. See, e.g., [Blecher and Le Merdy 2004, Section 1.3] for these. 
A hereditary subalgebra, or HSA, in an operator algebra $A$ is an approximately unital subalgebra with $D A D \subset D$. See [Blecher et al. 2008] for their basic theory. The support projection of an HSA in $A$ is the identity of its bidual, viewed within $A^{* *}$.

We write $\mathfrak{r}_{A}=\left\{x \in A: x+x^{*} \geq 0\right\}$, and call these the real positive elements. This space may be defined purely internally without using the "star", as the accretive elements, which have several purely metric definitions; see, e.g., [Blecher 2015, Lemma 2.4]. Also $\mathfrak{r}_{A}$ is the closure of the positive real multiples of $\mathfrak{F}_{A}=\{a \in A$ : $\|1-a\| \leq 1\}$ [Blecher and Read 2013]. Read's theorem states that any operator algebra with cai has a real positive cai; see [Blecher 2013] for a proof of this. Since $M_{n}(A)^{* *} \cong M_{n}\left(A^{* *}\right)$ [Blecher and Le Merdy 2004, Theorem 1.4.11], we have that $\mathfrak{r}_{M_{n}\left(A^{* *}\right)}$ is the weak-* closure of $\mathfrak{r}_{M_{n}(A)}$, and $\mathfrak{r}_{M_{n}(A)}=M_{n}(A) \cap \mathfrak{r}_{M_{n}\left(A^{* *}\right)}$ for each $n$.

A linear map $T: A \rightarrow B$ between operator algebras or unital operator spaces is real positive if $T\left(\mathfrak{r}_{A}\right) \subset \mathfrak{r}_{B}$. It is real completely positive, or RCP for short, if $T_{n}$ is real positive on $M_{n}(A)$ for all $n \in \mathbb{N}$. One may also define these maps in terms of the set $\mathfrak{F}_{A}$ above, as in [Blecher and Read 2013], but the definitions are shown to be equivalent in [Bearden et al. 2014, Section 2]. From the latter reference: a linear map $T: A \rightarrow B(H)$ on an approximately unital operator algebra or unital operator space $A$ is RCP if and only if $T$ has a completely positive (in the usual sense) extension $\tilde{T}: C^{*}(A) \rightarrow B(H)$. Here $C^{*}(A)$ is a $C^{*}$-algebra generated by $A$. We call this the generalized Arveson extension theorem. Thus real complete positivity on $A$ is equivalent to $P$ extending to a completely positive map on a containing $C^{*}$-algebra. A unital completely contractive map on a unital operator space is RCP, since it extends to a completely contractive map on a containing unital $C^{*}$-algebra, and such maps are completely positive, as we said above.

A ternary ring of operators, or TRO, is a subspace $Z$ of $B(K, H)$ such that $Z Z^{*} Z \subset Z$. A $W T R O$ is a weak-* closed TRO. The second dual of a TRO is a WTRO; see [Blecher and Le Merdy 2004, Chapter 8] for this and the next several facts. We write $L(Z)$ for the linking $C^{*}$-algebra of a TRO; this has "four corners": $Z Z^{*}, Z, Z^{*}$, and $Z^{*} Z$. Here $Z Z^{*}$ is the closure of the linear span of products $z w^{*}$ with $z, w \in Z$, and similarly for $Z^{*} Z$. One gets a similar von Neumann algebra for WTROs. A ternary morphism on a TRO $Z$ is a linear map $T$ such that $T\left(x y^{*} z\right)=T(x) T(y)^{*} T(z)$ for all $x, y, z \in Z$. A tripotent is an element $u \in Z$ such that $u u^{*} u=u$. We order tripotents by $u \leq v$ if and only if $u v^{*} u=u$. This turns out to be equivalent to $u=v u^{*} u$, or to $u=u u^{*} v$, and implies that $u^{*} u \leq v^{*} v$ and $u u^{*} \leq v v^{*}$ [Battaglia 1991]. If $x \in \operatorname{Ball}(Z)$, define $u(x)$ to be the weak-* limit of the sequence $\left(x\left(x^{*} x\right)^{n}\right)$ in $Z^{* *}$. This is the largest tripotent in $B^{* *}$ satisfying $v v^{*} x=v$ [Edwards and Rüttimann 1996]. If $x \geq 0$ or if $u(x)$ is a projection, then $u(x)$ is also the weak- $*$ limit of powers $x^{n}$ as $n \rightarrow \infty$; see, e.g., [Blecher and Neal 2012b; Blecher 2013].

We will say that an idempotent linear $P: X \rightarrow X$ is a symmetric projection if 
$\|I-2 P\| \leq 1$, and completely symmetric one if $\|I-2 P\|_{\mathrm{cb}} \leq 1$. This is related to the notion of $u$-ideal [Godefroy et al. 1993], but we will not need anything from that theory. Such are automatically bicontractive or completely bicontractive, respectively. We say that $P$ is completely hermitian if $P$ is hermitian in $C B(X)$. Note that since $\exp ($ it $P)=I-P+e^{i t} P$ it follows that $P$ is completely hermitian if and only if $\left\|I-P+e^{i t} P\right\|_{\mathrm{cb}} \leq 1$ for all real $t$. This is essentially the notion of being (completely) bicircular. Clearly if $P$ is completely hermitian then it is completely symmetric. We will not discuss (completely) hermitian projections much in this paper; these seem much less interesting.

\section{Completely contractive projections on approximately unital operator algebras}

Looking at examples it becomes clear that projections on operator algebras with no kind of approximate identity can be very badly behaved. Hence we will say little in our paper about such algebras. However, it is worth mentioning that we can pick out a "good part" of such a projection. This is the content of our first result.

Proposition 2.1. Let $P: A \rightarrow A$ be a real completely positive completely contractive map (resp. projection) on an operator algebra A (possibly with no kind of approximate identity). There exists a largest approximately unital subalgebra $D$ of $A$, and it is an HSA (hereditary subalgebra) of A. Moreover, $P(D) \subset D$, and the restriction $P^{\prime}$ of $P$ to $D$ is a real completely positive completely contractive map (resp. projection) on D. In addition, $P^{\prime}$ is completely bicontractive (resp. completely symmetric) if $P$ has the same property.

Proof. By [Blecher and Read 2014, Corollary 2.2], $D=\mathfrak{r}_{A}-\mathfrak{r}_{A}$ is the largest approximately unital subalgebra of $A$. This algebra is written as $A_{H}$ there, and was first introduced in [Blecher and Read 2013, Section 4]. Clearly $P(D) \subset D$. The rest is obvious.

Remark. The last result is also true with the word "completely" removed throughout, with the same proof.

Remark. Letting $p$ be the support projection of the HSA $D$ above, if $P$ extends to a completely positive complete contraction on a containing $C^{*}$-algebra (as in our generalized Arveson extension theorem mentioned in the introduction; see also [Bearden et al. 2014, Theorem 2.6]), then one can show that $P^{* *}(p a)=p P(a)$ and $P^{* *}(a p)=P(a) p$ for $a \in A$. It follows that $P$ may be pictured as a $2 \times 2$ matrix with its "good part" above in the $(1,1)$ corner. However, in general it seems one can say little about the other corners; they can be quite messy. This is why we focus on algebras with approximate identities in our paper. 
Proposition 2.2. A real completely positive completely contractive map (resp. projection) on an approximately unital operator algebra A extends to a unital real completely positive completely contractive map (resp. projection) on the unitization $A^{1}$. (If $A$ is unital then we define $A^{1}=A \oplus^{\infty} \mathbb{C}$ here.)

Proof. Suppose that $P: A \rightarrow A \subset B(H)$ is the map. By [Bearden et al. 2014, Theorem 2.6], $P$ extends uniquely to a completely positive completely contractive map $C^{*}(A) \rightarrow B(H)$. By [Choi and Effros 1976, Lemma 3.9] it extends further to a unital completely positive map $C^{*}(A)+\mathbb{C} I_{H} \rightarrow B(H)$. The restriction of the latter map to $A+\mathbb{C} I_{H}$ may be viewed as a unital (real completely positive) completely contractive map on the unitization $A^{1} \rightarrow A^{1}$, and it is evidently a projection if $P$ was a projection.

The previous result gives a way to reduce to the unital case. However, this method does not seem to be helpful later in our paper when dealing with bicontractive or symmetric projections, and we will need a different reduction to the unital case.

Lemma 2.3. Let $P: A \rightarrow A$ be a real positive, contractive map on a unital operator algebra. Then $0 \leq P(1) \leq 1$.

Proof. The restriction of $P$ to $\Delta(A)=A \cap A^{*}$ is real positive. Hence it is positive by the proof of [Bearden et al. 2014, Theorem 2.4]. So $0 \leq P(1) \leq 1$.

Lemma 2.4. Suppose that $E$ is a completely contractive completely positive projection on an operator system $X$. Then the range of $E$, with its usual matrix norms, is an operator system with matrix cones $E_{n}\left(M_{n}(X)_{+}\right)=M_{n}(X)_{+} \cap \operatorname{Ran}\left(E_{n}\right)$, and unit $E(1)$.

Proof. We will use the Choi-Effros characterization of operator systems [1977]. Because $\operatorname{Ran}\left(E_{n}\right)$ is a $*$-subspace of $M_{n}(X)$, with the inherited cone from $M_{n}(X)_{+}$, it is a partially ordered, matrix ordered, Archimedean $*$-vector space with proper cones. If $x=x^{*}$ there exists a positive scalar $t$ with $-t 1 \leq x \leq t 1$, so that $-t E(1) \leq x \leq t E(1)$. So $E(1)$ is an order unit. If $x \in \operatorname{Ran}(E)$ with $\|x\|_{X} \leq 1$ then

$$
\left[\begin{array}{cc}
1 & x \\
x^{*} & 1
\end{array}\right] \geq 0
$$

in $M_{2}(X)$. Applying $E_{2}$, we deduce that

$$
\left[\begin{array}{cc}
E(1) & x \\
x^{*} & E(1)
\end{array}\right] \geq 0
$$

so that the norm of $x$ is $\leq 1$ in the new order unit norm; see [Choi and Effros 1977, p. 179]. Conversely, if the last norm is $\leq 1$, or equivalently if the last centered equation holds, then it is a simple exercise in operator theory that $\|x\|_{X} \leq 1$, since $\|E(1)\| \leq 1$ and $E(1) \geq 0$. Thus the order unit norm coincides with the old norm. 
The computation is similar at each matrix level. By the Choi-Effros characterization of operator systems, $(\operatorname{Ran}(E), E(1))$ is an operator system with the given matrix cones, and the order-unit matrix norms are the usual norms.

The following generalization of the Choi-Effros result referred to in the introduction solves the completely contractive projection problem in the category of approximately unital operator algebras and real completely positive projections. We remark that the case when also $P\left(1_{A}\right)=1_{A}$ is implicit in the proof of [Blecher and Le Merdy 2004, Corollary 4.2.9].

Theorem 2.5. Let $A$ be an approximately unital operator algebra, and $P: A \rightarrow A$ a completely contractive projection which is also real completely positive. Then the range $B=P(A)$ is an approximately unital operator algebra with product $P(x y)$. We have

$$
P(P(a) b)=P(P(a) P(b))=P(a P(b)), \quad a, b \in A .
$$

In particular $\left.P\left(P(1)^{n}\right)\right)=P(1)$ for all $n \in \mathbb{N}$, if $A$ is unital. With respect to the "multiplication" $P(x y)$, we conclude that $A$ is a bimodule over $B$, and $P$, when viewed as a map $A \rightarrow B$, is a B-bimodule map (with $B$ equipped with its new product). If $A$ is unital then $P(1)$ is the identity for the latter product. Moreover (not assuming $A$ is unital), $P$ extends to a completely positive completely contractive projection on the injective envelope $I(A)$.

Proof. We give two proofs, since they both use techniques the reader will need to be familiar with in the rest of our paper.

Set $B=P(A)$. Extend $P$ to a unital completely contractive projection $P^{1}$ on $A^{1}$ by Proposition 2.2. We may then use the proof of [Blecher and Le Merdy 2004, Corollary 4.2.9], which proceeds by extending $P$ to a unital (completely positive and) completely contractive projection $E$ on $I\left(A^{1}\right)$. It follows from the Choi-Effros result mentioned early in our introduction, that $\operatorname{Ran}(E)$ is a unital $C^{*}$-algebra with product $E(x y)$, and $B$ with product $P(x y)$ is a unital subalgebra of this $C^{*}$-algebra. We also have by the same Choi-Effros result (or its proof) that $E(E(a) b)=E(E(a) E(b))=E(a E(b))$ for all $a, b \in A$, giving the centered equation in the theorem statement. This gives the first several assertions of our theorem. Note that

$$
P\left(P\left(e_{t}\right) P(a)\right)=P\left(e_{t} P(a)\right) \rightarrow P(P(a))=P(a), \quad a \in A,
$$

if $\left(e_{t}\right)$ is a cai for $A$, and similarly on the right, so that $\left(P\left(e_{t}\right)\right)$ is a cai for $P(A)$ in its new product. That $A$ is actually a $B$-bimodule follows from the centered equation in the theorem statement; for example, because

$$
\begin{aligned}
P(P(P(a) P(b)) c) & =P(P(P(a) P(b)) P(c))=P(P(a) P(b) P(c)) \\
& =P(P(a) P(P(b) P(c)))=P(P(a) P(P(b) c)) .
\end{aligned}
$$


The centered equation in the theorem statement is just saying that $P$ is a $B$-bimodule map for the given products. The final assertion about extending to $I(A)$ is easy from the above in the case that $A$ is unital; the other case we will do below.

For the second proof, first suppose that $A$ is unital. Let $B=P(A)$, and set $X=A+A^{*}, Y=B+B^{*}$, and $v=P(1)$. By [Bearden et al. 2014, Theorem 2.6], $P$ extends uniquely to a completely positive completely contractive map $P^{\prime}$ on $X$. Since $X=A+A^{*}$ this map is uniquely determined; it must be $a_{1}+a_{2}^{*} \mapsto P\left(a_{1}\right)+P\left(a_{2}\right)^{*}$, a projection on $X$ with range $Y$. By Lemma $2.4(Y, v)$ is an operator system with positive cone $P\left(X_{+}\right)$. Let $j: Y \rightarrow X$ be the inclusion map. Then extend $P^{\prime}$ to a completely positive complete contraction $\tilde{P}: I(A) \rightarrow I(B)$ [loc. cit.]. Extend $j$ to a completely positive complete contraction $\tilde{j}: I(B) \rightarrow I(A)$ by Arveson's extension theorem [1969]. Then $\tilde{P} \circ \tilde{j}$ equals the identity map on $I(B)$ by rigidity of the injective envelope, since $P \circ j=I_{B}$. Thus $E=\tilde{j} \circ \tilde{P}$ is a completely positive completely contractive projection on $I(A)$ extending $P$. We deduce just as in the last paragraph that $\operatorname{Ran}(E)$ is a unital $C^{*}$-algebra with product $E(x y), B$ with product $P(x y)$ is a unital subalgebra of this $C^{*}$-algebra, and $P(P(a) b)=P(P(a) P(b))=P(a P(b))$.

Finally, if $A$ is nonunital but approximately unital, then $P^{* *}$ is a completely contractive projection on $A^{* *}$, which is also real positive by the proof of the main theorem in [Bearden et al. 2014, Section 2]. By the unital case, $P^{* *}(x y)$ is an operator algebra product on $P^{* *}\left(A^{* *}\right)$, with unit $v=P^{* *}(1)$. Hence by restriction $P(x y)$ is an operator algebra product on $P(A)$, and the centered equation in the theorem statement holds on $A$, as does the assertions about bimodules. Note that $P^{* *}\left(A^{* *}\right)=(P(A))^{\perp \perp}$, so that $P^{* *}\left(A^{* *}\right) \cong P(A)^{* *}$. So $P(A)^{* *}$ is unital, and hence $P(A)$ is approximately unital (or this may be seen directly using the centered equation in the theorem statement). Also, since $v=P^{* *}(1)$ acts as an identity on $P(A)$ in the new product, we can identify $P(A)+\mathbb{C} v$, as a unital operator space, with the unitization of $P(A)$ with its new operator algebra product. Then the restriction $r$ of $P^{* *}$ to $A+\mathbb{C} 1_{A^{* *}}$ can be viewed as a real completely positive completely contractive projection on the unitization $A^{1}$. By the last paragraph, $r$ extends to a completely positive completely contractive projection on $I\left(A^{1}\right)$. However, $I\left(A^{1}\right)=I(A)$ by, e.g., [Blecher and Le Merdy 2004, Corollary 4.2.8].

Remark. Thus the category of approximately unital operator algebras and real completely positive projections forms a projectively stable category in the sense of Friedman and Russo; see [Neal and Russo 2011, p. 295-296] and, e.g., [Friedman and Russo 1985]. Namely, if $\mathcal{B}$ is the category of Banach spaces with morphisms being the contractive projections, a subcategory $\mathcal{S}$ of $\mathcal{B}$ is projectively stable if $\mathcal{S}$ is closed under images of morphisms. That is, for an object $E$ and morphism $\varphi: E \rightarrow E$ in $\mathcal{S}$, the image $\varphi(E)$ is again an object in $\mathcal{S}$ (although not necessarily a subobject with respect to the full structure of objects in $\mathcal{S}$ ). For example, the subcategory of 
unital $C^{*}$-algebras and completely positive unital projections is projectively stable, by the theorem of Choi and Effros used earlier. Other projectively stable categories are listed in the last references; e.g., the subcategory of TROs and completely contractive projections is projectively stable by Youngson's theorem [1983]. In the cited pages of [Neal and Russo 2011] the concept of a projectively rigid category is discussed. The associated question for us would be if the preduals of dual operator algebras are projectively (completely) rigid in their sense. However, the answer to this is in the negative, since the category of Banach or operator spaces is not projectively (completely) rigid, and then one can play the $\mathcal{U}(X)$ trick (described above Proposition 3.3 below) to answer the operator algebra question.

Remark. If $A$ is unital and $C$ is the $C^{*}$-subalgebra of $I(A)$ generated by $P(A)$, then the map $\tilde{P}$ in the proof restricts to a $*$-homomorphism from $C$ onto $C_{\mathrm{e}}^{*}(B)$, the latter viewed as a subalgebra of $I(B)$ (or as a $C^{*}$-subalgebra of the space $\operatorname{Ran}(E)$ in the proof, with its "Choi-Effros product"). See, e.g., [Blecher and Le Merdy 2004, Theorem 1.3.14(3)].

Lemma 2.6. Let $A$ be a unital operator algebra, and let $P: A \rightarrow A$ be a contractive projection, such that $\operatorname{Ran}(P)$ contains an orthogonal projection $q$ with $P(A)=q P(A) q$. Then $q=P\left(1_{A}\right)$.

Proof. We have $\|q \pm(1-q)\| \leq 1$ so that $\|q \pm P(1-q)\| \leq 1$. Since $P(A)=q P(A) q$, and $q$ is an extreme point of the unit ball of $q A q$ (the identity is an extreme point of the unit ball of any unital Banach algebra), $P(1-q)=0$. Thus $P(1)=q$.

The following reduction to the case of unital maps works under a certain condition which will be seen to be automatic in the setting found in the next sections of the paper.

Proposition 2.7. Let $A$ be an approximately unital operator algebra, and $P: A \rightarrow A$ a completely contractive projection. Then $\operatorname{Ran}\left(P^{* *}\right)$ contains an orthogonal projection $q$ such that $P(A)=q P(A) q$ if and only if $P^{* *}(1)$ is a projection. In this case, $q=P^{* *}(1)$ and $\operatorname{Ran}(P)$ is an approximately unital operator algebra with product $P(x y)$, and its bidual has identity $q$. Also, $P$ is real completely positive, all the conclusions of Theorem 2.5 hold, $q$ is an open projection for $A^{* *}$ in the sense of [Blecher et al. 2008], and

$$
P(a)=q P(a) q=P^{* *}(q a q), \quad a \in A
$$

(and we can replace $P^{* *}$ by $P$ here if $A$ is unital). Hence, $P(A)=q P(A) q=$ $P^{* *}(q A q)$, and $P$ splits as the sum of the zero map on $q^{\perp} A+A q^{\perp}+q^{\perp} A q^{\perp}$, and a real completely positive completely contractive projection $P^{\prime}$ on $q A q$ with range equal to $P(A)$. This projection $P^{\prime}$ on $q A q$ is unital if $A$ is unital. 
Proof. Let $Q=P^{* *}$, a completely contractive projection on $A^{* *}$. We can replace $Q$ by $P$ below if $A$ is unital. If $P(A)=q P(A) q$ for a projection $q$ then $Q\left(A^{* *}\right)=q Q\left(A^{* *}\right) q$ by standard weak-* approximation arguments, so by the lemma, $Q(1)=q$. Conversely, suppose that $Q(1)=q$ is a projection. Then $Q\left(q^{\perp}\right)=0$. Note that $\operatorname{Ran}(Q)$ is a unital operator space (in $q A^{* *} q$ ). So $Q$, and hence also $P$, is real completely positive by [Bearden et al. 2014, Lemma 2.2], since it extends by, e.g., [Blecher and Le Merdy 2004, Lemma 1.3.6] to a completely positive unital map from $X+X^{*}$ onto $Y+Y^{*}$, where $X=A^{* *}$ and $Y=Q\left(A^{* *}\right)$. By extending $Q$ further to a completely positive completely contractive map on a containing $C^{*}$-algebra, and using the Kadison-Schwarz inequality,

$$
Q\left(a q^{\perp}\right)^{*} Q\left(a q^{\perp}\right) \leq Q\left(q^{\perp} a^{*} a q^{\perp}\right) \leq Q\left(q^{\perp}\right)=0, \quad a \in \operatorname{Ball}\left(A^{* *}\right) .
$$

Thus $Q(a)=Q(a q)$ for all $a \in A^{* *}$, and similarly $Q(a)=Q(q a)$. Also $Q(q)^{2}=$ $Q(q)$, and so $P(A)=Q(q A q)=q P(A) q$ by Choi's multiplicative domain trick. (The latter is usually stated for unital maps, but the general case may be reduced to this using [Choi and Effros 1976, Lemma 3.9].)

The rest follows from Theorem 2.5 and its proof, with the exception of $q$ being an open projection for $A^{* *}$. To see this, if $\left(e_{t}\right)$ is a cai for $P(A)$ with its new product then using some of the facts here and in Theorem 2.5, we have $e_{t}=P\left(e_{t} q\right)=P\left(e_{t}\right) q \rightarrow q$ in the weak-* topology.

Remark. Note that even a completely contractive completely positive projection on a unital $C^{*}$-algebra need not have $P(1)$ a projection. To see this, choose a norm-1 element $x \neq 1$ in $A_{+}$and a state $\varphi$ with $\varphi(x)=1$, and consider $P=\varphi(\cdot) x$.

Remark. Unfortunately the projection $q$ here need not be central, even if $P$ is completely bicontractive. See the next example.

Example 2.8. Consider the canonical projection of the upper triangular matrices $A$ onto $\mathbb{C} E_{11}$. This is a real completely positive completely bicontractive projection (which is also completely bicontractive, completely hermitian, etc.), but it does not extend to a positive bicontractive projection on its $C^{*}$-envelope (or injective envelope) $M_{2}$. In this case note that $A+A^{*}=C^{*}(A)=C_{\mathrm{e}}^{*}(A)=I(A)$. On the positive side, the range of this projection is a subalgebra of $A$.

Corollary 2.9. Let $A$ be an approximately unital operator algebra with an approximately unital subalgebra $B$ which is the range of a completely contractive projection $P$ on A. Then $P$ is real completely positive, and all the conclusions of Theorem 2.5 hold. Hence $P$ is a conditional expectation: $P(a) b=P(a b)$ and $b P(a)=P(b a)$ for all $b \in B=P(A)$ and $a \in A$. It follows that $\left(P\left(e_{t}\right)\right)$ is a cai for $B$ for any cai $\left(e_{t}\right)$ of $A$.

Proof. Consider $P^{* *}$, a completely contractive projection on $A^{* *}$ with range $B^{* *}$. Of course $B^{* *}$ has an identity of norm-1 as we said in the introduction. By 
Proposition 2.7, $P^{* *}$ is real completely positive, and hence so is its restriction $P$. The remaining assertions follow, e.g., from Proposition 2.7, except for the last assertion, which is an easy consequence of the second to last assertion.

The last result, which may be seen as a converse to Theorem 2.5, generalizes the fact from [Blecher and Le Merdy 2004] mentioned at the start of the introduction. We showed in [Blecher 2004] that this is all false with the word "completely" removed; however, see [Lau and Loy 2008] for some later variants valid for certain Banach algebras.

We will need the following results later. If $P: M \rightarrow M$ is a unital completely contractive projection on a von Neumann algebra, there exists a support projection $e$, the perp of the supremum of all projections in $\operatorname{Ker}(P)$, as in [Effros and Størmer 1979, p. 129]. We have $e \in P(M)^{\prime}$, and $P(x)=P(e x)=P(x e)$ for all $x \in M$, and if $x \in M_{+}$then $P(x)=0$ if and only if $x e=0$ if and only if $e x=0$; see, e.g., around Lemma 1.2 in that reference. Following the idea in the proof of part (3) of that lemma, we have:

Proposition 2.10. Let $P: M \rightarrow M$ a weak-* continuous unital completely contractive projection on a von Neumann algebra $M$. Let e be the support projection of $P$ on $M$ discussed above. Let $N$ be the von Neumann algebra generated by $P(M)$. Then $P(x) e=e P(x) e=e x e=x e$ for all $x \in N$.

Proof. For $n=0,1, \ldots$, let $A_{n}$ be the span of products of $2^{n}$ elements from $P(M)$. Then $A_{n}$ is a unital $*$-subspace of $M$. Suppose that $e P(x) e=e x e$ for all $x \in A_{n}$. Then for such $x$, set $z=e\left(P\left(x^{*} x\right)-x^{*} e x\right) e$. Following the steps in the proof of [Effros and Størmer 1979, Lemma 1.2(3)] with minor modifications, we have $P(z)=0$ and $z \geq 0$, so that by the facts above the present proposition we obtain $z=e z e=0$ and $e P\left(x^{*} x\right) e=e x^{*} x e$. By the polarization identity, $e P\left(y^{*} x\right) e=e y^{*} x e$ for $x, y \in A_{n}$. So $e P(x) e=e x e$ for all $x \in A_{n+1}$, and hence for all $x \in N$.

Corollary 2.11. Let $P: A \rightarrow A$ be a unital completely contractive projection on an operator algebra. If $P(A)$ generates $A$ as an operator algebra, then $(I-P)(A)=$ $\operatorname{Ker}(P)$ is an ideal in $A$. In any case, if $D$ is the closed algebra generated by $P(A)$ then $(I-P)(D)$ is an ideal in $D$.

Proof. We may assume that $P(A)$ generates $A$. As above we extend $P$ to a unital completely contractive projection $\tilde{P}$ on a $C^{*}$-algebra $B$, which may be taken to be $I(A)$. The second adjoint of this is a weak-* continuous unital completely contractive projection on a von Neumann algebra $M$, and we continue to write this projection as $\tilde{P}$. Let $\tilde{P}$ also denote the restriction of the latter projection to the von Neumann algebra $N$ generated by $P(A)$ inside $M$. If $x \in(I-P)(A)$, then $\tilde{P}(x)=0$, and so by Proposition 2.10 we have $e x=x e=0$. Thus $x \in e^{\perp} M e^{\perp}$ (and is also in $e^{\perp} N e^{\perp}$ ). So for $y \in A$ we have $P(x y)=P($ exy $)=0$, and similarly $y x \in(I-P)(A)$, so the latter is an ideal. 
For later use we record that if $P: A \rightarrow A$ is a unital completely contractive projection on an operator algebra, then in the language of the last proof,

$$
D \cap e^{\perp} M e^{\perp}=D \cap e^{\perp} N e^{\perp}=\operatorname{Ker}\left(\left.P\right|_{D}\right)=(I-P)(D) .
$$

Indeed since we said above Proposition 2.10 that $P(x)=P($ exe $)$ for any $x \in M$, we have $D \cap e^{\perp} M e^{\perp} \subset \operatorname{Ker}\left(\left.P\right|_{D}\right)$. Moreover, if $d \in D$ with $P(d)=0$ then the argument in the last proof shows that $d \in D \cap e^{\perp} N e^{\perp} \subset D \cap e^{\perp} M e^{\perp}$. So

$$
D \cap e^{\perp} M e^{\perp}=D \cap e^{\perp} N e^{\perp}=\operatorname{Ker}\left(\left.P\right|_{D}\right) .
$$

\section{The symmetric projection problem and the bicontractive projection problem}

It turns out that the variant of the bicontractive projection problem for symmetric projections works out perfectly. This is the question of characterizing (completely) symmetric projections in the categories we are interested in, and their ranges. Notice that if $P: X \rightarrow X$ is a projection on a normed space and if we let $\theta=2 P-I$, so that $P=\frac{1}{2}(I+\theta)$, then $\operatorname{Ran}(P)$ is exactly the set of fixed points of $\theta$, and $\theta \circ \theta=I$. Note too that $\theta$ is contractive if $P$ is symmetric. From the latter facts we deduce that $\theta$ is a bijective isometry whose inverse is itself. Also $\theta(1)=1$ if $X$ is a unital algebra and $P(1)=1$. Applying the same argument at each matrix level we see:

Lemma 3.1. A projection $P: X \rightarrow X$ on an operator space is completely symmetric (resp. symmetric) if and only if $\theta=2 P-I$ is a complete isometry (resp. an isometry), and in this case $\theta$ is also a surjection. If $X$ is also an algebra (resp. Jordan algebra) and if $\theta$ is a homomorphism (resp. Jordan homomorphism), then the range of $P$ is a subalgebra (resp. Jordan subalgebra).

Proof. For the last part, $\operatorname{Ran}(P)$ is exactly the fixed points of $\theta$.

Thus the (completely) symmetric projection problem is in some sense a special case of the (complete) isometry problem: namely characterizing the linear (complete) isometries between the objects in our category. That is, the key to solving the (completely) symmetric projection problem is proving a Banach-Stonetype theorem in our category. The original Banach-Stone theorem characterizes unital isometries between $C(K)$ spaces, and in particular shows that such are *-isomorphisms. Putting this together with the last assertion of the last lemma, we see that one of the hoped-for conclusions of the (completely) symmetric projection problem, and by extension the (completely) bicontractive projection problem, is that the range of the projection is a subalgebra. We will also show in the completely symmetric case that if $A$ is unital or approximately unital then so is $P(A)$. 
Let us examine what this all looks like in a $C^{*}$-algebra, where as predicted in the last paragraph, much hinges on the known Banach-Stone-type theorem for $C^{*}$-algebras, due mainly to Kadison. The following is essentially well known (see, e.g., [Friedman and Russo 1984; Størmer 1982]), but we do not know of a reference which has all of these assertions, or is in the formulation we give.

Theorem 3.2. If $P: A \rightarrow A$ is a projection on a $C^{*}$-algebra $A$, then $P$ is bicontractive if and only if $P$ is symmetric. Then $P$ is bicontractive and completely positive if and only if there exists a central projection $q \in M(A)$, such that $P=0$ on $q^{\perp} A$, and there exists a period- $2 *$-automorphism $\theta$ of $q A$ so that $P=\frac{1}{2}(I+\theta)$ on $q A$.

Proof. Clearly symmetric projections are bicontractive. Conversely, if $P$ is bicontractive, then by [Friedman and Russo 1984, Theorem 2], $\theta=2 P-I_{A}$ is a linear surjective isometric selfmap of $A$ preserving the Jordan triple product and satisfying $\theta \circ \theta=I_{A}$ and $P=\frac{1}{2}\left(I_{A}+\theta\right)$. So $P$ is symmetric. If also $P$ is positive then $P$, and hence also $\theta$, is $*$-linear. Let $Q$ be the extension of $P$ to the second dual.

Suppose that $\theta: A \rightarrow B$ is a linear isometric surjection between $C^{*}$-algebras. By a result of Kadison [1951], $u=\theta^{* *}(1)$ is unitary in $B^{* *}$. Suppose now further that $\theta$ is $*$-linear. Then $u$ is selfadjoint, and $u \theta^{* *}(\cdot)$ is a unital isometry, so selfadjoint. Thus $u \theta(a)=\theta\left(a^{*}\right)^{*} u=\theta(a) u$, for $a \in A$, so $u$ is central. If $a \in A_{\mathrm{sa}}$ then

$$
u \theta\left(a^{2}\right)=(u \theta(a))^{2}=\theta(a)^{2} \in B,
$$

since $u \theta(\cdot)$ is a Jordan morphism. Thus $u B=u \theta(A) \subset B$. So $u \in M(B)$.

Returning to our situation, let $q=Q(1)$. This is a central projection in $M(A)$, since $u=2 q-1$. Since $Q\left(q^{\perp}\right)=0$, if $a \in \operatorname{Ball}(A)_{+}$then

$$
P\left(q^{\perp} a\right)=P\left(q^{\perp} a q^{\perp}\right) \leq Q\left(q^{\perp}\right)=0,
$$

and so $P=0$ on $q^{\perp} A$. Also, since $\theta(q)=q$ and $\theta$ preserves Jordan triple products, it follows that $P(q a)=\frac{1}{2}(q a+\theta(q a))=q P(a)$. Thus, $P(q A)=q P(A)$, and the restriction of $P$ to $q A$ is a unital bicontractive positive projection on a unital $C^{*}$-algebra. Also $\theta(q a)=q \theta(a)$ for $a \in A$, as we had above, so $\theta(q A)=q A$. Hence $\theta^{\prime}=\left.\theta\right|_{q A}$ is a unital isometric isomorphism of $q A$.

Remark. The example $P(x)=\frac{1}{2}\left(x+x^{T}\right)$ on $M_{2}$ shows the necessity of the completely positive hypothesis in the part it pertains to. Note in this example $P$ is positive and contractive, and $I-P$ is completely contractive.

We will generalize the bulk of the last result and its proof in Theorem 3.7.

We next show that unlike in the $C^{*}$-algebra case, for projections on operator algebras (completely) bicontractive is not the same as (completely) symmetric. Also, these both also differ from the notion of (completely) hermitian. We recall that any operator space $X \subset B(H)$ may be unitized to become an operator algebra 
as follows. We define

$$
\mathcal{U}(X)=\left\{\left[\begin{array}{cc}
\lambda_{1} I_{H} & x \\
0 & \lambda_{2} I_{H}
\end{array}\right]: x \in X, \lambda_{1}, \lambda_{2} \in \mathbb{C}\right\} \subset B(H \oplus H) .
$$

By definition, $\mathcal{U}(X)$ may be regarded as a subspace of the Paulsen system; see [Blecher and Le Merdy 2004, 1.3.14 and 2.2.10] or [Paulsen 2002]. It follows from Paulsen's lemma [op. cit., Lemma 8.1] or [Blecher and Le Merdy 2004, Lemma 1.3.15] that if $v: X \rightarrow X$ is a linear contraction (resp. complete contraction), then the mapping $\theta_{v}$ on $\mathcal{U}(X)$ defined by

$$
\theta_{v}\left(\left[\begin{array}{cc}
\lambda_{1} & x \\
0 & \lambda_{2}
\end{array}\right]\right)=\left[\begin{array}{cc}
\lambda_{1} & v(x) \\
0 & \lambda_{2}
\end{array}\right], \quad x \in X, \lambda_{1}, \lambda_{2} \in \mathbb{C},
$$

is a contractive (resp. completely contractive) homomorphism.

Proposition 3.3. Suppose that $X$ is an operator space, and that $P: X \rightarrow X$ is a linear idempotent map. Then $P$ is completely contractive (resp. completely bicontractive, completely symmetric, completely hermitian) if and only if the induced map $\tilde{P}: \mathcal{U}(X) \rightarrow \mathcal{U}(X)$ is a real completely positive and completely contractive (resp. completely bicontractive, completely symmetric, completely hermitian) projection. In particular these hold (and with the word "completely" removed everywhere if one wishes), if $P: X \rightarrow X$ is a linear idempotent map on a Banach space, when we give $X$ its minimal or maximal operator space structure; see, e.g., [Blecher and Le Merdy 2004; Paulsen 2002].

Proof. If $P$ is completely contractive then by Paulsen's lemma referred to above, the unique $*$-linear unital extension of $\tilde{P}$ to $\mathcal{U}(X)+\mathcal{U}(X)^{*}$ is completely contractive and completely positive. Thus by [Bearden et al. 2014, Section 2], $\tilde{P}$ is real completely positive. Clearly $\tilde{P}$ is a projection. Conversely, if $\tilde{P}$ is completely contractive then so is $P$. We note that $I-\tilde{P}$ annihilates the diagonal, and acts as $I-P$ in the $(1,2)$ entry. Thus $I-P$ is completely contractive if and only if $I-\tilde{P}$ is completely contractive. Also note that $2 \tilde{P}-I$ does nothing to the diagonal, and acts as $2 P-I$

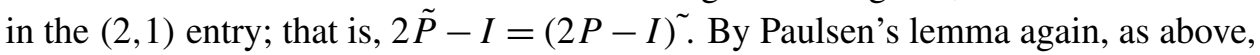
$2 \tilde{P}-I$ is completely contractive if and only if $2 P-I$ is completely contractive. Finally, $I-\tilde{P}+e^{i t} \tilde{P}$ multiplies each of the two diagonal entries by $e^{i t}$, and acts as $I-P+e^{i t} P$ in the $(1,2)$ entry. Multiplying by $e^{-i t}$, we see again by Paulsen's lemma that this is completely contractive if and only if $P$ is completely hermitian.

For the last assertion, if $P$ is contractive (resp. bicontractive, symmetric, hermitian), then it is completely contractive (resp. bicontractive, symmetric, hermitian) on $X$ with its minimal or maximal operator space structure by, e.g., [Blecher and Le Merdy 2004, 1.10 and 1.12]. We may then apply the case in the previous paragraph to obtain the result. Conversely, if $\tilde{P}$ is contractive (resp. bicontractive, etc.), then so is $P$ since $P$ is the $(1,2)$ corner of $\tilde{P}$. The rest is clear. 
The previous result provides examples of real completely positive unital completely bicontractive (resp. completely contractive, completely symmetric) projections which are not completely symmetric (resp. completely bicontractive, completely hermitian). As we said, this is in contrast to the $C^{*}$-algebra case where (complete) bicontractivity is equivalent to being (completely) symmetric [Størmer 1982; Friedman and Russo 1984].

Example 3.4. A more specific example of a real completely positive unital completely bicontractive projection on an operator algebra which is not symmetric arises by the last construction, from the following explicit completely bicontractive projection which is not completely symmetric. Let $Y$ be $\mathbb{R}^{2}$, the latter viewed as a real Banach space whose unit ball is the unit ball of $\ell_{2}^{\infty}$ in the first quadrant and the unit ball of $\ell_{2}^{1}$ in the second quadrant. Let $X$ be the standard complexification of $Y$. Take $P: X \rightarrow X$ to be the usual complexification of the projection onto the first coordinate on $Y$. We thank Asvald and Vegard Lima for this example, which is a bicontractive projection on a Banach space which is not symmetric. Giving $X$ its minimal or maximal operator space structure makes $X$ an operator space, and makes $P$ (by, e.g., [Blecher and Le Merdy 2004, 1.10 or 1.12]) a completely bicontractive projection which is not symmetric. Hence, by Proposition 3.3, we get a real completely positive unital completely bicontractive projection on an operator algebra which is not symmetric.

Example 2.8 shows that one cannot extend completely symmetric real completely positive projections to a positive bicontractive projection on a containing $C^{*}$-algebra. Things are much better if $P$ is a unital map:

Lemma 3.5. Let $A$ be a unital operator algebra, and let $P: A \rightarrow A$ be a completely symmetric unital projection. Then $P$ is real completely positive, and the range of $P$ is a subalgebra of $A$. Moreover, $P$ extends to a completely symmetric unital projection on $C_{\mathrm{e}}^{*}(A)($ or on $I(A))$.

Proof. Any completely contractive unital map on $A$ is real completely positive as we said in the introduction. Let $\theta=2 P-I$, so that $P=\frac{1}{2}(I+\theta)$. By Lemma 3.1, $\theta$ is a unital complete isometry. So in fact $\theta$ is a homomorphism, by the Banach-Stone theorem for operator algebras [Blecher and Le Merdy 2004]. This implies, as we said in Lemma 3.1, that $\operatorname{Ran}(P)$ is a subalgebra of $A$. We can extend $\theta$ uniquely to a unital *-isomorphism $\pi: C_{\mathrm{e}}^{*}(A) \rightarrow C_{\mathrm{e}}^{*}(A)$, with $\pi \circ \pi=I$, and it follows that $\tilde{P}=\frac{1}{2}(I+\pi)$ is a completely symmetric extension of $P$. It is also completely positive.

Similarly, since one may extend $\pi$ further to a unital $*$-automorphism of $I(A)$, there is a completely symmetric unital projection on $I(A)$ extending $P$.

Lemma 3.6. Let $A$ be a unital operator algebra, and let $P: A \rightarrow A$ be a completely contractive real positive projection which is bicontractive. Then $P(1)=q$ is a 
projection (not necessarily central), and all the conclusions of Theorem 2.5 and Proposition 2.7 hold. Also, there exists a unital completely bicontractive (real completely positive) projection $P^{\prime}: q A q \rightarrow q A q$ such that $P$ is the zero map on $q^{\perp} A+A q^{\perp}$ and $P=P^{\prime}$ on $q A q$. We have $\operatorname{Ran}(P)=\operatorname{Ran}\left(P^{\prime}\right)$.

Proof. Suppose that $q=P(1)$. Then $q \geq 0$ by Lemma 2.3, so that the closed algebra $B$ generated by $q$ and 1 is a $C^{*}$-algebra. Note that $P\left(q^{n}\right)=q$ by Theorem 2.5 , so that $P(B) \subset B$. Let $Q=\left.P\right|_{B}$; this is a bicontractive projection on $B$, and it is positive by the proof of Lemma 2.3. By [Friedman and Russo 1984, Theorem 2] and its proof, $q=P(1)$ is a partial isometry in $B$, hence a projection. Therefore, all the conclusions of Proposition 2.7 and Theorem 2.5 hold. So $P(a)=q P(a) q=P(q a q)$ for $a \in A$, and $P(A)=q P(A) q=P(q A q)$, so $P$ splits as the sum of the zero map on $q^{\perp} A+A q^{\perp}$ and a unital projection $E$ on $q A q$.

Remark. We give a direct proof (which also can be tweaked to work only assuming that $P$ is a bicontractive real positive projection on $A$ [Blecher et al. $\geq 2016$ ]) that $P(1)$ is a projection in the case of the lemma above: Let $P(1)=q$. As we saw in Theorem 2.5, $P\left(q^{n}\right)=q$. Thus $P(u(q))=q$, (we defined $u(\cdot)$ in the introduction). Suppose that $c$ is a positive scalar with $c(q-u(q))$ of norm 1 . Then

$(I-P)(c(q-u(q))-u(q))=c(q-u(q))-u(q)-(c(q-q)-q)=(1+c)(q-u(q))$,

which has norm greater than 1 . By the contractivity of $I-P$ we have a contradiction, unless $q=u(q)$. So $q=P(1)$ is a projection.

The following is the solution to the symmetric projection problem in the category of approximately unital operator algebras.

Theorem 3.7. Let $A$ be an approximately unital operator algebra, and let $P: A \rightarrow A$ be a completely symmetric real completely positive projection. Then the range of $P$ is an approximately unital subalgebra of $A$. Moreover, $P^{* *}(1)=q$ is a projection in the multiplier algebra $M(A)$ (so is both open and closed).

Set $D=q A q$, a hereditary subalgebra of A containing $P(A)$. There exists a period-2 surjective completely isometric homomorphism $\theta: A \rightarrow A$ such that $\theta(q)=q$, so that $\theta$ restricts to a period-2 surjective completely isometric homomorphism $D \rightarrow D$. Also, $P$ is the zero map on $q^{\perp} A+A q^{\perp}+q^{\perp} A q^{\perp}$, and

$$
P=\frac{1}{2}(I+\theta) \text { on } D \text {. }
$$

In fact,

$$
P(a)=\frac{1}{2}(a+\theta(a)(2 q-1)), \quad a \in A .
$$

The range of $P$ is exactly the set of fixed points of $\left.\theta\right|_{D}$ in $D$.

Conversely, any map of the form in the last equation, for a period-2 surjective completely isometric homomorphism $\theta: A \rightarrow A$ and a projection $q \in M(A)$ with $\theta^{* *}(q)=q$, is a completely symmetric real completely positive projection. 
Proof. Applying Lemma 3.6 to $P^{* *}$, we see that $P^{* *}(1)=q$ is a projection, and all the conclusions of Proposition 2.7 and Theorem 2.5 are true for us. We will silently be using facts from these results below. In particular $q$ is an open projection, so it supports an approximately unital subalgebra $D$ of $A$ with $D^{\perp \perp}=q A^{* *} q$; see [Blecher et al. 2008]. Then $\theta=2 P-I$ is a linear completely isometric surjection on $A$ by Lemma 3.1. So by the Banach-Stone theorem for operator algebras [Blecher and Le Merdy 2004, Theorem 4.5.13], there exists a completely isometric surjective homomorphism $\pi: A \rightarrow A$ and a unitary $u$ with $u, u^{-1} \in M(A)$ with $\theta=\pi(\cdot) u$. We have

$$
\theta^{* *}(1)=2 P^{* *}(1)-1=2 q-1=\pi^{* *}(1) u=u,
$$

so that $u$ is a selfadjoint unitary (a symmetry), and $q \in M(A)$. So $q A q=D \subset A$. Since $P(A)=q P(A) q$, the range of $P$ is contained in $D$, and the range of $P$ is exactly the set of fixed points of $\theta$, which all lie in $D$. This implies that $\operatorname{Ran}(P)$ is a subalgebra of $A$. It is approximately unital and $P$ is real completely positive by Corollary 2.9.

We have $\theta^{* *}(q)=q$ and $\pi^{* *}(q)=\theta^{* *}(q) u=q u=q$. Then $2 P(a)-a=$ $\pi(a)(2 q-1)$ and $P(a)=\frac{1}{2}(a+\pi(a)(2 q-1))$. Indeed

$$
\pi(a)=(2 P(a)-a) u=(2 P(a)-a)(2 q-1)=2 P(a)-2 a q+a, \quad a \in A .
$$

From this, or otherwise, one sees that $\pi$ equals $\theta$ on $D$, and

$$
\pi(D)=(2 P-I)(D) \subset D .
$$

However, $D=\theta^{2}(D) \subset \theta(D)=\pi(D)$, so $\pi(D)=D$. This completes the main part of the theorem.

For the converse, note that $P(a)=\frac{1}{2}(a+\pi(a)(2 q-1))$ is clearly completely symmetric on $A$, and

$$
\begin{aligned}
P(P(a)) & =P\left(\frac{1}{2}(a+\pi(a)(2 q-1))\right) \\
& =\frac{1}{4}(a+2 \pi(a)(2 q-1)+\pi(\pi(a)(2 q-1))(2 q-1)=P(a),
\end{aligned}
$$

since $\pi$ is period $2, \pi^{* *}(q)=q$, and $2 q-1$ is a symmetry. We have

$$
P^{* *}(1)=\frac{1}{2}\left(1+\pi^{* *}(1)(2 q-1)\right)=q,
$$

so that $P$ is real completely positive by Proposition 2.7 .

It follows easily from the last theorem that a completely symmetric real completely positive projection $P$ on $A$ extends to a completely symmetric projection $\tilde{P}$ on the $C^{*}$-envelope of $A$. Moreover, $\tilde{P}(x)=\frac{1}{2}(a+\tilde{\pi}(a)(2 q-1))$, for a $*$-automorphism $\tilde{\pi}$. However, this extension will not in general be positive. 
In the work [Blecher et al. $\geq 2016$ ] in progress, we prove the Jordan algebra variant of the last result.

\section{The bicontractive projection problem}

The following could be compared with Corollary 2.11.

Lemma 4.1. Let $A$ be a unital operator algebra, and $P: A \rightarrow A$ a unital projection with $I-P$ completely contractive. Let $C=(I-P)(A)=\operatorname{Ker}(P)$. Then $C^{2} \subset P(A)$. We also have:

(1) $C$ is a subalgebra of $A$ if and only if $C^{2}=(0)$.

(2) If $P$ is completely bicontractive (or more generally, if $P(a P(b))=P(P(a) P(b)$ ) for $a, b \in A$ ) then $P(A) C+C P(A) \subset C$, and $C^{3} \subset C$.

(3) If the conditions in (1) hold, and if $P$ is completely contractive (or more generally, if $P(a P(b))=P(P(a) P(b))$ for $a, b \in A)$ then $C$ is actually an ideal in $A$.

(4) If $P(A) C+C P(A) \subset C$ (see (2)), then $\theta=2 P-I_{A}$ is a homomorphism if and only if $P(A)$ is a subalgebra of $A$, and then the range of $P$ is the set of fixed points of this automorphism $\theta$.

Proof. Of course $A=C+B$, where $B=P(A)$. By Youngson's result [1983] applied to an extension $Q$ of $I-P$ to a completely contractive projection on $I(A)$ (which exists by an easier variant of the proof in Theorem 2.5), we have $(I-P)(w z)=Q\left(w(I-P)(1)^{*} z\right)=0$ for $z, w \in C$. So $P(w z)=w z \in B$, and this is zero if the kernel is a subalgebra. In any case, $C^{2} \subset B$. Assuming that $P$ is completely contractive (or that $P(a P(b))=P(P(a) P(b))$ for $a, b \in A$, which is a weaker condition by, e.g., the fact in the first paragraph of our paper), if $z \in B$ and $w \in C$, then $P(w z)=P(P(w) z)=0$, so $w z \in C$. So $C B \subset C$ and similarly $B C \subset C$. Thus $C^{3} \subset B C \subset C$. This proves (2). If also $C$ is a subalgebra, then it is an ideal, proving (3).

For (4), we may decompose $A=C \oplus B$, where $1_{A} \in B=P(A)$, and we have the relations $C^{2} \subset B, C B+B C \subset C$. Using the latter it is a simple computation that the period-2 map $\theta: x+y \mapsto x-y$ for $x \in B, y \in C$ is a homomorphism (indeed an automorphism) on $A$ if and only if $P(A)$ is a subalgebra of $A$; clearly $P(A)$ is the set of fixed points of $\theta$.

Remark. Replacing $P$ by $I-P$ we see that if $P: A \rightarrow A$ is a completely contractive projection with $P(1)=0$, then $P(A)$ is a subalgebra if and only if $P(A)^{2}=(0)$.

Remark. The kernel of a bicontractive projection need not be a subalgebra. For example, consider $P(f)(x)=\frac{1}{2}(f(x)+f(-x))$ for $f \in C([-1,1])$. 
The following clarifies the unital version of the bicontractive projection problem in relation to the existence of an associated period-2 automorphism.

Corollary 4.2. If $P: A \rightarrow A$ is a unital idempotent on a unital operator algebra, let $\theta: A \rightarrow A$ be the associated linear period-2 automorphism $x+y \mapsto x-y$ for $x \in \operatorname{Ran}(P), y \in \operatorname{Ker}(P)$. Then $P$ is completely bicontractive if and only if $\|I \pm \theta\|_{\mathrm{cb}} \leq 2$. If these hold then the range of $P$ is a subalgebra if and only if $\theta$ is also a homomorphism, and then the range of $P$ is the set of fixed points of this automorphism $\theta$. Also, $P$ is completely symmetric if and only if $\theta$ is completely contractive.

Proof. The first and last assertions are obvious, and the second assertion follows from Lemma 4.1(4).

For us, the bicontractive projection problem is whether the range of a completely bicontractive real completely positive projection on an approximately unital operator algebra $A$ is an approximately unital subalgebra of $A$. This is not obvious, although there are easy counterexamples if one drops some of the hypotheses. For example, consider the projection $P$ on the upper triangular $2 \times 2$ matrices given by

$$
\left[\begin{array}{ll}
a & b \\
0 & c
\end{array}\right] \mapsto\left[\begin{array}{cc}
\frac{1}{2}(a-c) & 0 \\
0 & \frac{1}{2}(c-a)
\end{array}\right]
$$

This is completely contractive and extends to a completely contractive projection on the containing $C^{*}$-algebra, and can be shown to be bicontractive with $P(1)=0$, and its range is not a subalgebra.

We now show that the bicontractive projection problem can be reduced to the case that $A$ is unital and $P(1)=1$, by a sequence of three reductions. First, if $P: A \rightarrow A$ is a completely bicontractive real completely positive projection on an approximately unital operator algebra $A$, then $P^{* *}$ is a completely bicontractive real completely positive projection on a unital operator algebra. Thus, henceforth in this section and in the next, we assume that $A$ is unital. Second, Lemma 3.6 allows us to reduce further to the case that $P(1)=1$ : it asserted that $P(1)=q$ is a projection (not necessarily central), all the conclusions of Theorem 2.5 and Proposition 2.7 hold, and there exists a unital completely bicontractive (real completely positive) projection $P^{\prime}: q A q \rightarrow q A q$ such that $P$ is the zero map on $q^{\perp} A+A q^{\perp}, P=P^{\prime}$ on $q A q$, and $\operatorname{Ran}(P)=\operatorname{Ran}\left(P^{\prime}\right)$.

Then the third of our reductions of the completely bicontractive projection problem puts the problem in a standard position. Of course, $P(A)$ is a subalgebra if and only if $Q(D)$ is a subalgebra, where $D$ is the closed subalgebra of $A$ generated by $P(A)$, and $Q=\left.P\right|_{D}$, which is a completely bicontractive unital projection on $D$. That is, we may as well replace $A$ by the closed subalgebra generated by $P(A)$. 
Thus by these three steps above, we have reduced the completely bicontractive projection problem on approximately unital operator algebras to the standard position of a unital projection on a unital operator algebra, whose range generates $A$. In this situation we obtain the following structural result.

Corollary 4.3. Let $P$ be a completely bicontractive unital projection on a unital operator algebra $A$. Let $D$ be the algebra generated by $P(A)$. Then $(I-P)(D)=$ $\operatorname{Ker}\left(\left.P\right|_{D}\right)$ is an ideal in $D$ and the product of any two elements in this ideal is zero.

Proof. We saw at the end of Section 2 that $(I-P)(D)=\operatorname{Ker}\left(\left.P\right|_{D}\right)$ is an ideal in $D$, and in the notation there it equals $D \cap e^{\perp} M e^{\perp}$. Since $D \cap e^{\perp} M e^{\perp}$ is a subalgebra of $D$ the result follows from Lemma 4.1.

Remark. Note that $(I-P)(D) \subset e^{\perp} A$, but $P(D)$ is not a subset of $e A$.

The above shows that we can also solve the bicontractive projection problem in the affirmative for real completely positive completely bicontractive projections $P$ on a unital operator algebra $A$ such that the closed algebra generated by $A$ is semiprime (that is, it has no nontrivial square-zero ideals):

Corollary 4.4. Let $P: A \rightarrow A$ be a real completely positive completely bicontractive projection on a unital operator algebra. If $A$ is an operator algebra containing no nonzero nilpotents, then $P(A)$ is a subalgebra of $A$. Also if the closed algebra $D$ generated by $P(A)$ is semiprime, then $P(A)$ is a subalgebra of $A$.

Proof. By the second reduction above, we may assume that $P$ is unital. By Corollary 4.3, $(I-P)(D)$ is an ideal in $D$ with square zero, and so is $(0)$ in these cases. So $P(A)=P(D)=D$, a subalgebra.

For the subcategory of uniform algebras (that is, closed subalgebras of $C(K)$, for compact $K$ ) which are unital or approximately unital, the bicontractive projection problem coincides with the symmetric projection problem, and again there is a complete solution:

Theorem 4.5. Let $P: A \rightarrow A$ be a real positive bicontractive projection on a uniform algebra $A$, and suppose that $A$ is unital or approximately unital. Then $P$ is (completely) symmetric, and so we have all the conclusions of Theorem 3.7. In particular, $P(A)$ is a subalgebra of $A$, and $P$ is a conditional expectation.

Proof. Here bicontractive projections are the same as completely bicontractive projections [Blecher and Le Merdy 2004, 1.10]. By the obvious variant of the usual proof that positive maps into a $C(K)$ space are completely positive, we have that real positive maps into a uniform algebra are real completely positive. By the first two reductions described above we can assume that $A$ and $P$ are unital. We also know that $B=P(A)$ is a subalgebra by Corollary 4.4, since, e.g., nonzero nilpotents cannot exist in a function algebra. Thus by Corollary 4.2 the map $\theta(x+y)=x-y$ 
described there is an algebra automorphism of $A$, hence a (completely) isometric isomorphism (since norm equals spectral radius). So $P=\frac{1}{2}(I+\theta)$ is (completely) symmetric, and Theorem 3.7 applies.

Remark. The idea in the last proof that $\theta$ is automatically isometric, since it is an algebraic automorphism of a uniform algebra, and that this implies that $P$ is symmetric, was found together with Joel Feinstein after submission.

By, e.g., Corollary 4.2, to find a counterexample to the conjecture that all (completely real positive) completely bicontractive unital projections have range which is a subalgebra, we need a unital operator algebra $D=C \oplus E$ where

$$
C \neq(0), \quad C^{2}=0, \quad C E+E C \subset C, \quad 1_{D} \in E, \quad \text { and } E \text { generates } D,
$$

so in particular $E^{2}$ is not a subset of $E$, and with the projection maps onto $C$ and $E$ completely contractive. This is easy in a Banach algebra; one may equip $\ell_{3}^{1}$, with the standard basis identified with symbols $1, a, b$ satisfying relations like $b^{2}=0, a^{2}=b$, etc. (setting $C=\{b\}, E=\operatorname{Span}\{1, a\}$ ). To find an operator algebra example, we make a general construction.

Example 4.6. Let $V$ be a closed subspace of $B(H) \oplus B(H)$, viewed as elements of $B\left(H^{(2)}\right)$ supported on the $(1,1)$ and $(2,2)$ entries. Write $v_{1}$ and $v_{2}$ for the two "parts" of an element $v \in V$. Let $C$ be the closed span of $\left\{v_{1} w_{2}: v, w \in V\right\}$, and we will assume that $C \neq(0)$. Let $B$ be the set of elements of $B\left(H^{(3)}\right)$ of the form

$$
\left[\begin{array}{ccc}
\lambda I & v_{1} & c \\
0 & \lambda I & v_{2} \\
0 & 0 & \lambda I
\end{array}\right], \quad \lambda \in \mathbb{C}, v \in V, c \in C .
$$

Then the copy of $C$ is an ideal in $B$ with square zero, and it is generated by the copy of $V$ in $B$. If $E$ is the sum of this copy of $V$ and $\mathbb{C} I_{H^{(3)}}$, then all the conditions in the last paragraph needed for a counterexample hold, with the exception of the projection onto $E$ being completely contractive. We remark that one may also describe $B$ more abstractly as a set $B=\mathbb{C} 1+V+C$ in $B(K)$, where $V$ and $C$ are closed subspaces of $B(K)$ with the properties that

$$
(0) \neq C=V^{2}, \quad V^{3}=C^{2}=(0),
$$

plus one more condition ensuring that $p_{1} V(1-p)=(0)$, where $p_{1}$ is the left support projection of $C$ and $p$ is the left support projection of $C+V$. 
To fix the exception noted in the last paragraph, we consider the subalgebra $A=A(V)$ of $B\left(H^{(7)}\right)$ consisting of all elements of form

$$
\left[\begin{array}{ccccccc}
\lambda I & v_{1} & c & 0 & 0 & 0 & 0 \\
0 & \lambda I & v_{2} & 0 & 0 & 0 & 0 \\
0 & 0 & \lambda I & 0 & 0 & 0 & 0 \\
0 & 0 & 0 & \lambda I & 0 & 2 v_{1} & 0 \\
0 & 0 & 0 & 0 & \lambda I & 0 & 2 v_{2} \\
0 & 0 & 0 & 0 & 0 & \lambda I & 0 \\
0 & 0 & 0 & 0 & 0 & 0 & \lambda I
\end{array}\right], \quad \lambda \in \mathbb{C}, v \in V, c \in C .
$$

We will abusively write the square-zero ideal in $A$ consisting of the copy of $C$ again as $C$, and again let $E$ be the sum of $\mathbb{C} I_{H^{(7)}}$ and the isomorphic copy of $V$ in $A(V)$, so that $A=C \oplus E$. Here

$$
C \neq(0), \quad C^{2}=0, \quad C E+E C \subset C, \quad 1_{D} \in E, \quad \text { and } E \text { generates } A,
$$

as desired. The canonical projection map from $A$ onto $C$ is obviously completely contractive.

A particularly simple case is when $H$ is one-dimensional, so that $A \subset M_{7}$, and where $V=\mathbb{C I}_{2}$. This algebra is obviously essentially just (i.e., is completely isometrically isomorphic to) the subalgebra of $M_{5}$ of matrices

$$
\left[\begin{array}{ccccc}
\lambda & \nu & c & 0 & 0 \\
0 & \lambda & v & 0 & 0 \\
0 & 0 & \lambda & 0 & 0 \\
0 & 0 & 0 & \lambda & 2 v \\
0 & 0 & 0 & 0 & \lambda
\end{array}\right], \quad \lambda, v, c \in \mathbb{C},
$$

with the projection being replacing the $(1,3)$ entry by 0 . Thus $5 \times 5$ matrices of scalars will suffice to give an interesting example. However, we will need the more general construction later to produce a more specialized counterexample.

Consider the algebra $\mathcal{U}(V)$ constructed from $V$ as described in the paragraph above Proposition 3.3. We define $\mathcal{U}_{0}(V)$ to be the subalgebra of $\mathcal{U}(V)$ consisting of elements of $\mathcal{U}(V)$ with the two diagonal entries identical. It is a subalgebra of $B\left(H^{(4)}\right)$.

Lemma 4.7. In the situation of Example 4.6, the map $j: \mathcal{U}_{0}(V) \rightarrow B\left(H^{(3)}\right)$ given by

$$
\left[\begin{array}{cc}
\lambda I & v \\
0 & \lambda I
\end{array}\right] \mapsto\left[\begin{array}{ccc}
\lambda I & \frac{1}{2} v_{1} & 0 \\
0 & \lambda I & \frac{1}{2} v_{2} \\
0 & 0 & \lambda I
\end{array}\right]
$$

is completely contractive and unital. 
Proof. To prove that it is completely contractive simply notice that $j$ may be viewed as the composition of the canonical map $\mathcal{U}(V) \rightarrow \mathcal{U}(W)$, where $W$ is the copy of $V$ in the algebra $B$ above, and the map $M_{2}(B) \rightarrow B$ given by pre- and postmultiplying by $\left[\frac{1}{\sqrt{2}} I_{H^{(3)}} \frac{1}{\sqrt{2}} I_{H^{(3)}}\right]$ and its transpose.

Corollary 4.8. If $A=A(V)$ is the unital operator algebra above in $B\left(H^{(7)}\right)$ then the canonical projection $P: A \rightarrow A$ which replaces the $(1,3)$ entry by 0 , whose kernel is a nontrivial square-zero ideal $C$ generated by $P(A)$, is a (real completely positive) completely bicontractive and unital projection, but its range is not a subalgebra, and it need not even be a Jordan subalgebra. A particularly simple case is the algebra (completely isometrically isomorphic to the algebra of) of $5 \times 5$ scalar matrices described above Lemma 4.7.

Proof. In the $5 \times 5$ matrix example it is easily checked that $P(A)$ is not closed under squares, hence is not a Jordan subalgebra. It remains to prove that $P$ is completely contractive. However, $P$ is the composition of the canonical map $B\left(H^{(3)} \oplus H^{(4)}\right) \rightarrow B\left(H^{(4)}\right)$ restricted to $A$, and the map $x \mapsto j(x) \oplus x$ on $\mathcal{U}_{0}(V)$, where $j$ is as in Lemma 4.7 .

The following is another rather general condition under which the completely bicontractive projection problem is soluble. Indeed as we said in the introduction, all examples known to us of real completely positive completely bicontractive projections on unital operator algebras, whose range is a subalgebra, do satisfy the criterion in Theorem 4.9.

Theorem 4.9. Let $A$ be a unital operator algebra in a von Neumann algebra $M$ (which could be taken to be $B(H)$, or $I(A)^{* *}$ as above) and let $P: A \rightarrow A$ be a unital completely bicontractive projection. Let $D$ be the closed algebra generated by $P(A)$, and let $C=(I-P)(D)$.

Suppose further that $C P(A)^{*} \subset \overline{M C}^{w *}$, the weak-* closed left ideal in $M$ generated by $C$. (This is equivalent to saying that the left support projection of $P(A) C^{*}$ is dominated by the right support projection of $C$.) Alternatively, assume that $P(A)^{*} C$ is contained in the weak-* closed right ideal in $M$ generated by $C$ (or equivalently that the right support projection of $C^{*} P(A)$ is dominated by the left support projection of $C$ ). Then $P(A)$ is a subalgebra of $A$.

Proof. We assume the left ideal condition; the other case is similar and left to the reader (or can be seen by looking at the opposite algebra $A^{\mathrm{op}}$ ). By replacing $A$ by $D$ we may assume that $P(A)$ generates $A$. Suppose that $M$ is a von Neumann algebra on a Hilbert space $H$. We set $p_{1}=\bigvee_{z \in C} r(z) r(z)^{*}$ to be the left support projection of $C$ and $p_{2}=\bigvee_{z \in C} r(z)^{*} r(z)$ to be the right support projection of $C$. Note that $z p_{1}=0$ for $z \in C$, which implies that $p_{2} p_{1}=0$. Let $p=p_{1}+p_{2}$, a 
projection. Our hypothesis is equivalent to saying that

$$
C P(A)^{*}=C P(A)^{*} p_{2} .
$$

We may write

$$
M=(1-p) M(1-p)+(1-p) M p+p M(1-p)+p M p .
$$

Thus $M$ may be pictured as the direct sum of a von Neumann algebra with four corners (thus having a $2 \times 2$ matrix form). Let $y \in P(A)$. Then $y C \subset C \subset p M$, so that $(1-p) y p_{1}=0$. On the other hand, by hypothesis, $p_{2} y^{*}(1-p)=p_{2} y^{*} p_{2}(1-p)=0$. Thus $(1-p) y p_{2}=0$ and so $(1-p) y p=0$. Therefore,

$$
y=(1-p) y(1-p)+p y(1-p)+p y p=y(1-p)+p y p .
$$

Furthermore $p_{2} y C \subset p_{2} C=p_{2} p_{1} C=(0)$, and so $p_{2} y p_{1}=0$. And by hypothesis, $p_{1} y p_{2}=p_{1}\left(p_{2} y^{*}\right)^{*}=p_{1}\left(p_{2} y^{*} p_{2}\right)^{*}=0$. So

$$
p_{1} P(A) p_{2}=(0) .
$$

Thus

$$
y=y(1-p)+p_{1} y p_{1}+p_{2} y p_{2} .
$$

If also $x \in P(A)$ then $x=x(1-p)+p_{1} x p_{1}+p_{2} x p_{2}$, and so

$$
x y=x(1-p) y(1-p)+p_{1} x p_{1} y p_{1}+p_{2} x p_{2} y p_{2},
$$

and so again $p_{1} x y p_{2}=0$. Since $C=(I-P)(A)$ we see that

$$
(I-P)(x y)=p_{1}(I-P)(x y) p_{2}=p_{1} x y p_{2}-p_{1} P(x y) p_{2}=0,
$$

so that $x y=P(x y) \in P(A)$. So $P(A)$ is a subalgebra.

Remark. If $C P(A)^{*} \subset[B C]$ where $B=C_{\mathrm{e}}^{*}(A)$ then the first hypothesis in the previous result holds.

Remark. If $A$ is the counterexample algebra of $5 \times 5$ scalar matrices described above Lemma 4.7, it is very illustrative to compute the various associated objects of interest in our paper. We leave the details to the reader as an exercise. Here $C^{*}(A)=$ $C_{\mathrm{e}}^{*}(A)=I(A)=M_{3} \oplus M_{2} \subset M_{5}$. If $P$ is the projection in that example, namely the map that replaces the $(1,3)$ entry with 0 , then $C^{*}(P(A))=I(P(A))=0 \oplus M_{2} \subset M_{5}$. A completely contractive completely positive projection $\tilde{P}$ on $C^{*}(A)=I(A)$ that extends $P$ is the map

$$
x \oplus\left[\begin{array}{ll}
a & b \\
c & d
\end{array}\right] \mapsto\left[\begin{array}{ccccc}
\frac{1}{2}(a+d) & \frac{1}{2} b & 0 & 0 & 0 \\
\frac{1}{2} c & \frac{1}{2}(a+d) & \frac{1}{2} b & 0 & 0 \\
0 & \frac{1}{2} c & \frac{1}{2}(a+d) & 0 & 0 \\
0 & 0 & 0 & a & b \\
0 & 0 & 0 & c & d
\end{array}\right], \quad x \in M_{3} .
$$


To see that this is completely contractive is a tiny modification of the proof of Lemma 4.7. A completely contractive projection extending $I-P$ is the projection onto the $(1,3)$ coordinate. The support projection of $\tilde{P}$ defined just before Proposition 2.10 is $e=0 \oplus I_{2}$, which has complement $r=I_{3} \oplus 0$. The projections $p_{1}, p_{2}, p$ from the proof of Theorem 4.9 are $p_{1}=E_{11}, p_{2}=E_{33}, p=E_{11}+E_{33}$, and $1-p=E_{22}+E_{44}+E_{55}$. Also, $r-p=E_{22}$. Note that $C P(A)^{*} p_{2} \neq C P(A)^{*}$, of course. Indeed this example is an excellent illustration of what is going on in the proof of Theorem 4.9. Note that if we change the definition of $A$ by replacing either the $(2,3)$ entry or the $(3,2)$ entry then the hypotheses of Theorem 4.9 are satisfied.

Examining why the general example described in Example 4.6 does not satisfy the hypotheses of Theorem 4.9 is illustrative: it is not hard to see that if it did then $v_{1} w_{2} z_{2}^{*}=0$ for all $v, w, z \in V$. However, if $0 \neq \sum_{k=1}^{n} v_{1}^{k} w_{2}^{k} \in C$ then we obtain the contradiction

$$
0 \neq\left(\sum_{k=1}^{n} v_{1}^{k} w_{2}^{k}\right)\left(\sum_{k=1}^{n} v_{1}^{k} w_{2}^{k}\right)^{*}=0 .
$$

It would be interesting to investigate other conditions that might imply that $P(A)$ is a subalgebra, particularly when in the standard position (namely $P: A \rightarrow A$ is a unital completely bicontractive projection whose range generates $A$ as an operator algebra). Some which might be worth investigating are if the algebra $C$ in Theorem 4.9 is a maximal ideal in $A$, or if $C$ contains the radical of $A$. Note that any one of these conditions rules out our counterexamples above.

\section{Another condition}

We now look at another condition on a completely contractive projection $P$ which is automatic for bicontractive projections in the $C^{*}$-algebra case, namely that the induced projection on $\operatorname{Re}(A)$ is bicontractive. We will not assume that the induced projection on $\operatorname{Re}(A)$ has completely contractive complementary projection $I-P$. We are not able to solve the problem yet, but have made some progress towards the solution.

Lemma 5.1. Let $A$ be a unital operator algebra, and let $P: A \rightarrow A$ be a unital completely contractive projection such that the induced projection on $\operatorname{Re}(A)$ is bicontractive. We also write $P$ for an extension to a unital completely contractive weak-* continuous projection on the von Neumann algebra $B^{* *}$, where $B$ is a $C^{*}$-algebra containing $A$ as a unital subalgebra (see the argument in the proof of Corollary 2.11). Let e be the support projection of $P$ on $B$ as in [Effros and Størmer 1979, p. 129]. If $x \in A \cap e^{\perp} B e^{\perp}$ and $x=a+i b$ with $a=a^{*}, b=b^{*}$, then $\left\|a_{+}\right\|=\left\|a_{-}\right\|=\left\|b_{+}\right\|=\left\|b_{-}\right\|$.

Proof. Suppose that $\|a\| \leq 1$. By the Kadison-Schwarz inequality,

$$
P(a)^{*} P(a) \leq P\left(a^{*} a\right)=P\left(e^{\perp} a^{*} a e^{\perp}\right) \leq P\left(e^{\perp}\right)=0 .
$$


So $P(a)=0$, and similarly $P(b)=0$. Suppose that $\left\|a_{+}\right\|>\left\|a_{-}\right\|$. Then by the spectral theorem for $a$, there exists $\epsilon>0$ with

$$
\|a-\epsilon 1\|=\left\|a_{+}-a_{-}-\epsilon 1\right\|<\left\|a_{+}-a_{-}\right\|=\|a\| .
$$

Thus $\|a-\epsilon 1\|<\|(I-P)(a-\epsilon 1)\|$, a contradiction. So $\left\|a_{+}\right\| \leq\left\|a_{-}\right\|$. A similar argument shows that $\left\|a_{-}\right\| \leq\left\|a_{+}\right\|$; therefore, $\left\|a_{+}\right\|=\left\|a_{-}\right\|$. Similarly (or by replacing $x$ by $i x),\left\|b_{+}\right\|=\left\|b_{-}\right\|$.

Let $y=\operatorname{Re}\left(2 x-x^{2}\right)$. The last paragraph shows that $\|y\|=\left\|y_{+}\right\|=\left\|y_{-}\right\|$. Now assume that $\|a\|=1 \geq\|b\|$. So $\left\|a_{ \pm}\right\|=1$. Let $\psi$ be a state with $\psi\left(a_{-}\right)=1$. Then $\psi\left(a_{+}\right)=0$ or else $\psi(|a|)=\psi\left(a_{+}\right)+\psi\left(a_{-}\right)>1$, which is impossible. By standard arguments these imply that $\psi\left(a_{-}^{2}\right)=1$ and $\psi\left(a_{+}^{2}\right)=0$. Since

$$
y=2 a_{+}-2 a_{-}-\left(a_{+}^{2}+a_{-}^{2}\right)+\left(b_{+}^{2}+b_{-}^{2}\right),
$$

we have

$$
\psi(y)=-3+\psi\left(b_{+}^{2}+b_{-}^{2}\right) .
$$

It is well known that for a selfadjoint operator $T=T_{+}-T_{-}=R-S$ with $R, S \geq 0$, we have $\left\|T_{+}\right\| \leq\|R\|$. Thus

$$
\|y\|=\left\|y_{+}\right\| \leq\left\|2 a_{+}-a_{+}^{2}+\left(b_{+}^{2}+b_{-}^{2}\right)\right\| \leq 2 .
$$

Since $\psi(y)=-3+\psi\left(b_{+}^{2}+b_{-}^{2}\right)$ we must have $\psi\left(b_{+}^{2}+b_{-}^{2}\right)=1$, so that $\left\|b^{2}\right\|=1=\|b\|$. Replacing $x$ by $i x$, we see that $\|a\|=\|b\|$.

Lemma 5.2. Let $A$ be a unital operator algebra, and let $P: A \rightarrow A$ be a unital completely contractive projection such that the induced projection on $\operatorname{Re}(A)$ is bicontractive. We also write $P$ for an extension to a unital completely contractive weak-* continuous projection on the von Neumann algebra $B^{* *}$, where $B$ is a $C^{*}$-algebra containing $A$ as a unital subalgebra (as in the last result). Let $e$ be the support projection of $P$ on $B$ as in [Effros and Størmer 1979, p. 129]. If $x \in A \cap e^{\perp} B e^{\perp}$ and $x=a+i b$ with $a=a^{*}, b=b^{*}$, and $\|a\|=1$, then $u(a)^{2}=u(b)^{2}$.

Proof. Since $b=b^{*}$, we know that $u(b)$ is a selfadjoint tripotent and $u(b)^{2}$ is a projection. It is well known that $u(x)^{*} u(x)=u\left(x^{*} x\right)$ (to see this, note that $x\left(x^{*} x\right)^{n} \rightarrow x u\left(x^{*} x\right)$, so that $x u\left(x^{*} x\right)=u(x)$ from which the relation is easy). Hence $u(b)^{2}=u\left(b^{2}\right)=u\left(b_{+}^{2}+b_{-}^{2}\right)$. As we saw in the last proof, if $\psi$ is a state with $\psi\left(a_{-}\right)=1$ then $\psi\left(b_{+}^{2}+b_{-}^{2}\right)=1$. Now $\psi\left(a_{-}\right)=1$ if and only if $\psi\left(u\left(a_{-}\right)\right)=1$, and $\psi\left(b_{+}^{2}+b_{-}^{2}\right)=1$ if and only if $\psi\left(u(b)^{2}\right)=1$; see [Edwards and Rüttimann 1996, Lemma 3.3(i)]. So $\left\{u\left(a_{-}\right)\right\}^{\prime} \cap S(B) \subset\left\{u(b)^{2}\right\}^{\prime} \cap S(B)$, where $S(B)$ is the state space and the "prime" is as in [op. cit.]. From this, as is well known (and simple to prove), we have that $u\left(a_{-}\right) \leq u(b)^{2}$. Similarly, $u\left(a_{+}\right) \leq u(b)^{2}$, so that $u\left(a_{-}\right)+u\left(a_{+}\right)=u(a)^{2} \leq u(b)^{2}$. Similarly, $u(b)^{2} \leq u(a)^{2}$, so $u(a)^{2}=u(b)^{2}$. 
Lemma 5.3. Let $A$ be a unital operator algebra, and let $P: A \rightarrow A$ be a unital completely contractive projection such that the induced projection on $\operatorname{Re}(A)$ is bicontractive. We also write $P$ for an extension to a unital completely contractive weak-* continuous projection on the von Neumann algebra $B^{* *}$, where $B$ is a $C^{*}$-algebra containing $A$ as a unital subalgebra (as in the last results). Let e be the support projection of $P$ on $B$ as in [Effros and Størmer 1979, p. 129]. Suppose that $x \in A \cap e^{\perp} B e^{\perp}$ has norm-1. Then $u(x)^{2}=0$ and $x=u(x)+y$ for some $y \in B^{* *}$ with $u(x) y=u(x) y^{*}=y u(x)=y^{*} u(x)=0$. Finally, $\|\operatorname{Re} x\|=\frac{1}{2}$.

Proof. Suppose that $x \in A \cap e^{\perp} B e^{\perp}$, and choose an angle $\theta$ so that $\left\|\operatorname{Re}\left(e^{i \theta} x\right)\right\|$ is maximized. By Lemma 5.1 this equals $\left\|\operatorname{Im}\left(e^{i \theta} x\right)\right\|$. Write $z=e^{i \theta} x=a+i b$ with $a=a^{*}, b=b^{*}$. Scale $z$ so that $\|a\|=1$ (so $\|b\|=1$ by Lemma 5.1). Write $a=u(a)+a_{\perp}$ and $b=u(b)+b_{\perp}$. Note that $u(a) a_{\perp}=u(a)(a-u(a))=0$, since $u(a) a=u(a)^{3} a=u(a)^{2}$. Similarly $a_{\perp} u(a)=0$, and $b_{\perp} u(b)=u(b) b_{\perp}=0$. Since $u(b)^{2}=u(a)^{2}$ by Lemma 5.2, we have $u(a) b_{\perp}=u(a) u(b)^{2} b_{\perp}=0$, and similarly $b_{\perp} u(a)=a_{\perp} u(b)=u(b) a_{\perp}=0$. Hence $a_{\perp}$ and $b_{\perp}$ are contractions by the orthogonality of $u(a)$ and $a_{\perp}$, and of $u(b)$ and $b_{\perp}$. Consider

$$
\frac{1+i}{\sqrt{2}}(a+i b)=\frac{a-b}{\sqrt{2}}+i \frac{a+b}{\sqrt{2}} .
$$

By the maximality property of $\theta$ we have $\|(a-b) / \sqrt{2}\|=\|(a+b) / \sqrt{2}\| \leq 1$. Now

$$
\frac{u(a)-u(b)}{\sqrt{2}}=u(a)^{2} \frac{a-b}{\sqrt{2}} \text { and } \frac{u(a)+u(b)}{\sqrt{2}}=u(a)^{2} \frac{a+b}{\sqrt{2}},
$$

so these are contractions. Squaring each of these we see that

$$
u(a)^{2}-\frac{1}{2}(u(a) u(b)+u(b) u(a)) \quad \text { and } \quad u(a)^{2}+\frac{1}{2}(u(a) u(b)+u(b) u(a))
$$

are contractions. Since $u(a)^{2}$ is a projection, hence an extreme point, we deduce that $u(a) u(b)+u(b) u(a)=0$, or $u(a) u(b)=-u(b) u(a)$. Using this, if $w_{1}=$ $\frac{1}{2}(u(a)+i u(b))$ then a simple computation shows that $w_{1} w_{1}^{*} w_{1}=w_{1}$, so that $w_{1}$ is a partial isometry. Let $w=z / 2$. Clearly $\|w\| \leq 1$, but now we see that

$$
1=\left\|w_{1}\right\|=\left\|u(a)^{2} w\right\| \leq\|w\| .
$$

So $\|w\|=1$ and $\|z\|=\|x\|=2$. This proves the last assertion of the theorem, since $\|\operatorname{Re}(x)\|=\|\operatorname{Im}(x)\| \leq 1$ by the maximality property of $\theta$, but they clearly cannot be strict contractions since $\|x\|=2$. So henceforth we may assume that $\theta=0$ and $z=x$.

Let $w_{2}=\frac{1}{2}\left(a_{\perp}+i b_{\perp}\right)$, so that $w=w_{1}+w_{2}$, and $w_{1} w_{2}=w_{2} w_{1}=w_{1} w_{2}^{*}=$ $w_{2}^{*} w_{1}=0$. Note that $u(w)=w_{1}+u\left(w_{2}\right) \neq 0$, and $u\left(e^{-i \theta} w\right)=e^{-i \theta} u(w)$, so $\|x\|=2$. Also $w w^{*}=w_{1} w_{1}^{*}+w_{2} w_{2}^{*}$. Suppose $\psi$ is a state with $\psi\left(w_{2} w_{2}^{*}\right)=1$. Then since $\left\|w w^{*}\right\| \leq 1$ we must have $\psi\left(w_{1} w_{1}^{*}\right)=0$, which forces $\psi\left(u(a)^{2}\right)=\psi\left(u(b)^{2}\right)=0$. 
Thus $\psi \notin\left\{u\left(a^{2}\right)\right\}^{\prime}=\left\{a^{2}\right\}^{\prime}$ [Edwards and Rüttimann 1996, Lemma 3.3(i)], so $\psi\left(a_{\perp}^{2}\right) \neq 1$ since $a^{2}=u\left(a^{2}\right)+a_{\perp}^{2}$. On the other hand,

$$
1=\psi\left(\frac{a_{\perp}^{2}}{4}+\frac{b_{\perp}^{2}}{4}+i\left(\frac{b_{\perp} a_{\perp}}{4}-\frac{a_{\perp} b_{\perp}}{4}\right)\right) .
$$

We deduce the contradiction that $\psi\left(a_{\perp}^{2}\right)=1$. This contradiction shows that $1-w_{2} w_{2}^{*}$ is strictly positive so that $u\left(w_{2} w_{2}^{*}\right)=0$. Hence $u\left(w w^{*}\right)=u\left(w_{1} w_{1}^{*}\right)=w_{1} w_{1}^{*}$, and so $u(w)=\lim _{n \rightarrow \infty}\left(w w^{*}\right)^{n} w=w_{1} w_{1}^{*} w=w_{1}$.

Finally, suppose that $x \in A \cap e^{\perp} B e^{\perp}$ has norm-1 (so that $x$ may be taken to be our previous $w)$. Then $u(x)^{2}=w_{1}^{2}=0$. That $x=u(x)+y$, where $u(x)$ is orthogonal to $y$ and $y^{*}$, follows because $w=u(w)+w_{2}$ and

$$
u(w) w_{2}=u(w) w_{2}^{*}=w_{2} u(w)=w_{2}^{*} u(w)=0,
$$

the latter because $u(w)$ is a linear combination of the selfadjoint $u(a), u(b)$, which are each orthogonal to $a_{\perp}$ and $b_{\perp}$.

Corollary 5.4. If the conditions of the previous lemmas hold and $A \cap e^{\perp} B e^{\perp}=(0)$, then $P(A)$ is a subalgebra of $A$.

Proof. For $x, y \in P(A)$ we have exye $=e P(x y) e$ by Proposition 2.10. Thus $x y-P(x y) \in e^{\perp} B^{* *} e^{\perp} \cap A=(0)$, showing that $P(A)$ is closed under products.

Corollary 5.5. If the conditions of the previous lemmas hold and $B$ is commutative, then $A \cap e^{\perp} B e^{\perp}=(0)$ and $P(A)$ is a subalgebra of $A$.

Proof. By the proof of Lemma 5.3, if $x \in A \cap e^{\perp} B e^{\perp}$ and $e^{i \theta} x=a+i b$ with $a=a^{*}, b=b^{*}$, and $\|a\|=1$, we obtained $u(a) u(b)=-u(b) u(a)=0$ and $u(a)=u(a) u(b)^{2}=0$. This is impossible since $\|a\|=1$, so $A \cap e^{\perp} B e^{\perp}=(0)$. Then apply Corollary 5.4.

As in Section 4, to show $P(A)$ is a subalgebra of $A$, we may replace $A$ by $D$, the closed algebra generated by $P(A)$. After this is done, in the previous lemmas $A \cap e^{\perp} B e^{\perp}$ becomes $(I-P)(D)$.

Theorem 5.6. Let $P$ be a unital completely contractive projection on A such that $I-P$ is contractive on $\operatorname{Re}(A)$. Suppose that $A$ is a subalgebra of $M_{N}$ for some $N \in \mathbb{N}$ and let $D$ be the closed algebra generated by $P(A)$. Then every element of $(I-P)(D)$ is nilpotent. Furthermore, if $D$ is semisimple then the range of $P$ is a subalgebra of $A$.

Proof. Note that $(I-P)(D)$ is an ideal of $D$ by Corollary 2.11. Suppose that $x \in(I-P)(D)$ has norm-1 and is not nilpotent. Set $y_{1}=x$. By Lemma 5.3, $x=u(x)+x$, where $u(x)^{2}=0$ and $x_{1} \perp u(x)$. Furthermore $x^{2}=\left(x_{1}\right)^{2}$ lies in $D$ and $x^{2} \perp u(x)$. Similarly, since $x^{2} \neq 0$ we set $y_{2}=x^{2} /\left\|x^{2}\right\|$. Then $y_{2}=u\left(y_{2}\right)+\left(y_{2}\right)$, where $u\left(y_{2}\right)$ and $\left(y_{2}\right)$, are perpendicular to each other, and $u\left(y_{2}\right)$ is perpendicular 
to $u(x)$. (This is because $u\left(y_{2}\right)$ is a limit of products beginning and ending with $x^{2}$, and, e.g., $x^{2} u(x)=\left(x_{1}\right)^{2} u(x)=0$.) Continuing in this way, we obtain an infinite sequence of norm- 1 elements $y_{k}$ such that $u\left(y_{n}\right) \perp u\left(y_{k}\right)$ for $k \leq n$. It is well known that $u(y) \neq 0$ if $\|y\|=1$. This contradicts finite-dimensionality. So $x$ is nilpotent. Since $(I-P)(D)$ is an ideal of $D$ consisting of nilpotents, it follows that it lies in the Jacobson radical of $D$. Thus if $D$ is semisimple then $(I-P)(D)=(0)$, so that $P(A)=D$ as before.

For the following we no longer assume $A$ is finite-dimensional but retain the other assumptions of the above theorem.

Lemma 5.7. Let $P$ be a unital completely contractive projection on $A$ such that $I-P$ is contractive on $\operatorname{Re}(A)$, and let $D$ be the closed algebra generated by $P(A)$. If $x \in(I-P)(D)$ and $\|x\|=1$, then $\left\|x^{2^{n}}\right\| \leq 2 / 2^{2^{n}}$. Also, $x$ is quasiregular (that is, quasi-invertible).

Proof. Let $x=a+i b$ as in previous lemmas. By Lemmas 5.1 and 5.2, $\|a\|=\|b\|=\frac{1}{2}$. Hence $\left\|\operatorname{Re}\left(x^{2}\right)\right\|=\left\|a^{2}-b^{2}\right\| \leq \frac{1}{4}$. Again from Lemma 5.1, $\left\|x^{2}\right\| \leq \frac{1}{2}$. The first result now follows by considering normalizations of further powers of 2 , and using mathematical induction. It is easily seen that

$$
\sum_{k=1}^{\infty}\left\|x^{k}\right\| \leq 1+\sum_{m=1}^{\infty} 2^{m}\left\|x^{2^{m}}\right\| \leq 1+\sum_{m=1}^{\infty} 2^{m} \frac{2}{2^{2^{m}}}<\infty .
$$

It follows that $\sum_{k=1}^{\infty} x^{k}$ converges, so that $1-x$ is invertible.

Remark. It is still open whether the ideal $(I-P)(D)$ above consists entirely of quasiregular elements. If this is the case, then the above Theorem 5.6 holds for arbitrary unital operator algebras. Note too that the assertion about quasiregulars in Lemma 5.7 does follow from Lemma 5.1. That result shows that the ideal $(I-P)(D)$ in $D$ has no nonzero real positive elements (for, in the language of that result, if $a_{-}=0$ then $\left.a_{+}=b_{+}=b_{-}=0\right)$. The ideas in the proof of [Blecher and Read 2013, Corollary 6.9] then also show that if $x \in \operatorname{Ball}((I-P)(D))$ then $x$ is quasiregular.

\section{Jordan morphisms and Jordan subalgebras of operator algebras}

We recall that a Jordan homomorphism $T: A \rightarrow B$ is a linear map satisfying $T(a b+b a)=T(a) T(b)+T(b) T(a)$ for $a, b \in A$, or equivalently, that $T\left(a^{2}\right)=$ $T(a)^{2}$ for all $a \in A$ (the equivalence follows by applying $T$ to $\left.(a+b)^{2}\right)$. By a Jordan operator algebra we shall simply mean a norm-closed Jordan subalgebra A of an operator algebra, namely a norm-closed subspace closed under the Jordan product $\frac{1}{2}(a b+b a)$, or equivalently with $a^{2} \in A$ for all $a \in A$ (that is, $A$ is closed under squares). 
It is natural to ask if the completely bicontractive algebra problem studied in Section 4 becomes simpler if the range of the projection $P: A \rightarrow A$ is also a Jordan subalgebra (that is, $\left.P(a)^{2} \in P(A)\right)$ for all $a \in A$. We next dispose of this question:

Example 6.1. Let $y=E_{21} \oplus E_{12} \in M_{4}$, and let

$$
x=\left[\begin{array}{rrrr}
0 & 0 & 0 & 1 \\
0 & 0 & -1 & 0 \\
0 & 0 & 0 & 0 \\
0 & 0 & 0 & 0
\end{array}\right] .
$$

Then $x y=-y x$, so that if $F$ is the span of $x$ and $y$ then $F$ is closed under squares. However, $F$ is not an algebra since $x y \notin F$. Let $V=\left\{z \oplus z \in M_{8}: z \in F\right\}$, and form the algebra $A=A(V)$ described in Example 4.6. By Corollary 4.8 the canonical projection $P: A \rightarrow A$ which replaces the $(1,3)$ entry of a matrix in $A(V)$ by 0 , is a (real completely positive) completely bicontractive and unital projection, but its range is not a subalgebra. However, its range is a Jordan subalgebra; $P(A)$ is closed under squares since $z^{2}=0$ for $z \in F$. Thus, the completely bicontractive algebra problem does not become simpler if the range of the projection $P: A \rightarrow A$ is also a Jordan subalgebra.

The following variant of the Banach-Stone theorem for $C^{*}$-algebras will be evident to "JB-experts".

Lemma 6.2. Let $A$ be a unital $C^{*}$-algebra, and $T: A \rightarrow B(H)$ a unital complete isometry such that $T(A)$ is closed under taking squares (thus, $T(A)$ is a Jordan algebra). Then $T(A)$ is a $C^{*}$-subalgebra of $B(H)$, and $T$ is a*-homomorphism.

Proof. Since such $T$ is necessarily $*$-linear as we said in the introduction, $T(A)$ is a $\mathrm{JB}^{*}$-algebra, hence a selfadjoint $\mathrm{JB}^{*}$-triple; see, e.g., [Cabrera García and Rodríguez Palacios 2014]. By the theory of JB*-triples $T$ is a Jordan homomorphism. (Two other proofs of this: look at the selfadjoint part and use the fact that isometries in that category are Jordan homomorphisms [Isidro and Rodríguez-Palacios 1995]; or it can be deduced using the $C^{*}$-envelope as in the next proof). In particular for each $x \in A_{\text {sa }}$ we have $T\left(x^{2}\right)=T(x)^{2}$, so by Choi's multiplicative domain result (see, e.g., [Blecher and Le Merdy 2004, Proposition 1.3.11]) we have $T(x y)=T(x) T(y)$ for all $y \in A$. So $T$ is a homomorphism and $T(A)$ is a $C^{*}$-subalgebra.

It is natural to ask if the analogous result is true for operator algebras. That is, if $B$ is a closed unital Jordan subalgebra of an operator algebra $A$, and if $B$ is unitally and linearly completely isometric to another unital operator algebra, then is $B$ actually a subalgebra of $A$ ? If the algebra is also commutative this is true and follows from the next result. 
Lemma 6.3. Let $A$ be a unital operator algebra, and let $T: A \rightarrow B$ be a unital complete isometry onto a unital Jordan operator algebra. Then $T$ is a Jordan homomorphism, and $T\left(a^{n}\right)=T(a)^{n}$ for every $n \in \mathbb{N}$ and $a \in A$.

Proof. Note that $T(a)^{3}$ is the Jordan product of $T(a)$ and $T(a)^{2}$, so $T(A)$ is closed under cubes. Similarly it is closed under every power. By the property of the $C^{*}$-envelope mentioned in the introduction, there exists a $*$-homomorphism $\pi: C^{*}(T(A)) \rightarrow C_{\mathrm{e}}^{*}(A)$ with $\pi \circ T=I_{A}$. So $a^{n}=\pi\left(T(a)^{n}\right)=\pi\left(T\left(a^{n}\right)\right)$. Since $\left.\pi\right|_{T(A)}$ is one-to-one, the results follow.

Remark. In the proof of the last result one could have instead used [Arazy and Solel 1990, Corollary 2.8].

We now answer the question above Lemma 6.3 in the negative:

Example 6.4. Let $P: A \rightarrow A$ be a completely contractive projection on an operator algebra $A$ on $H$ whose kernel is an ideal $I$; see, e.g., Corollary 2.11 or Lemma 4.1. Then it is known that $B=A / I$ is an operator algebra [Blecher and Le Merdy 2004, Proposition 2.3.4], and the induced map $\tilde{P}: B \rightarrow P(A)$ is a completely isometric isomorphism, and $\tilde{P}$ will be unital if $A$ and $P$ are unital. If these hold, and in addition $P(A)$ is a Jordan subalgebra of $A$ which is not a subalgebra, then $T=\tilde{P}: B \rightarrow A \subset B(H)$ is a unital complete isometry such that $T(B)$ is closed under taking squares (thus, $T(B)$ is a unital Jordan subalgebra), but $T(B)$ is not a subalgebra, and $T$ is not an algebra homomorphism. In particular, we can take $A$ to be the algebra in Example 6.1.

We finish our paper with another Banach-Stone-type theorem for operator algebras:

Proposition 6.5. Suppose that $T: A \rightarrow B$ is a completely isometric surjection between approximately unital operator algebras. Then $T$ is real (completely) positive if and only if $T$ is an algebra homomorphism.

Proof. If $T$ is an algebra homomorphism then by Meyer's theorem [Blecher and Le Merdy 2004, Theorem 2.1.13], $T$ extends to a unital completely isometric surjection between the unitizations, which then extends by Wittstock's extension theorem to a unital completely contractive, hence completely positive, map on a generated $C^{*}$-algebra. So $T$ is real completely positive.

Conversely, suppose that $T$ is real positive. We may assume that $A$ and $B$ are unital, since $T^{* *}$ is a real positive completely isometric surjection between unital operator algebras. By the Banach-Stone theorem for operator algebras [op. cit., Theorem 4.5.13], there exists a completely isometric surjective homomorphism $\pi: A \rightarrow B$ and a unitary $u$ with $u, u^{-1} \in B$ with $T=\pi(\cdot) u$. The restriction of $T$ to $\mathbb{C} 1$ is real positive, hence positive; see [Bearden et al. 2014, Section 2]. Thus $u \geq 0$, and so $u=\left(u^{2}\right)^{1 / 2}=1$. Hence $T$ is an algebra homomorphism. 
Remark. One may also prove a limited version of this result for algebras with no kind of approximate identity by using the ideas in the proof of Proposition 2.1.

There is also a Jordan variant of the last result, a simple adaption of the main theorem in [Arazy and Solel 1990]. Here we just state the unital case; see [Blecher et al. $\geq 2016]$ for more on this topic.

Proposition 6.6. Suppose that $T: A \rightarrow B$ is an isometric surjection between unital Jordan operator algebras. Then $T$ is real positive if and only if $T$ is a Jordan algebra homomorphism.

Proof. If $T$ is a Jordan algebra homomorphism and $u=T\left(1_{A}\right)$ and $T(v)=1_{B}$ then $2 u=u 1_{B}+1_{B} u=2 T\left(1_{A} \cdot v\right)=2 T(v)=2 \cdot 1_{B}$. So $u=1_{B}$. However, a unital contractive map is real positive [Bearden et al. 2014, Section 2].

The converse follows by the same proof as for Proposition 6.5, but using the form of the Banach-Stone theorem for operator algebras in [Arazy and Solel 1990, Corollary 2.10]. By that result $T(1)$ is a unitary $u$ with $u, u^{-1} \in B$. Moreover $T(\cdot) u^{-1}$ is an isometric surjection onto $B$, so by the same result it is a Jordan homomorphism $\pi$. We have $T=\pi(\cdot) u$, and we finish as before.

\section{Acknowledgments}

Some of this material was presented at an AMS special session in January 2015 (Operator Algebras and Their Applications: A Tribute to Richard V. Kadison), as briefly surveyed in the article [Blecher 2015], which will appear in the proceedings of that meeting. Corollary 4.2 and part of Theorem 4.5 were first stated in that article, and later added to the present paper. We thank Joel Feinstein for a conversation during which we together arrived at an insight needed in these results. We are also indebted to the referee for suggesting that we consider the Jordan algebra version of the topics of this paper; this insight led to the work [Blecher et al. $\geq 2016$ ] in progress.

\section{References}

[Akemann 1970] C. A. Akemann, "Left ideal structure of $C^{*}$-algebras", J. Functional Analysis 6 (1970), 305-317. MR 0275177 Zbl 0199.45901

[Arazy and Solel 1990] J. Arazy and B. Solel, "Isometries of nonselfadjoint operator algebras", $J$. Funct. Anal. 90:2 (1990), 284-305. MR 1052336 Zbl 0713.46043

[Arveson 1969] W. B. Arveson, "Subalgebras of $C^{*}$-algebras", Acta Math. 123 (1969), 141-224. MR 0253059 Zbl 0194.15701

[Barton and Timoney 1986] T. Barton and R. M. Timoney, "Weak*-continuity of Jordan triple products and its applications”, Math. Scand. 59:2 (1986), 177-191. MR 884654

[Battaglia 1991] M. Battaglia, "Order theoretic type decomposition of JBW*-triples", Quart. J. Math. Oxford Ser. (2) 42:166 (1991), 129-147. MR 1107278 Zbl 0736.46052 
[Bearden et al. 2014] C. A. Bearden, D. P. Blecher, and S. Sharma, "On positivity and roots in operator algebras”, Integral Equations Oper. Theory 79:4 (2014), 555-566. MR 3231244 Zbl 1311.46051

[Blecher 2004] D. P. Blecher, “Are operator algebras Banach algebras?", pp. 53-58 in Banach algebras and their applications, edited by A. T.-M. Lau and V. Runde, Contemporary Mathematics 363, American Mathematical Society, Providence, RI, 2004. MR 2097949 Zbl 1078.46040

[Blecher 2013] D. P. Blecher, "Noncommutative peak interpolation revisited", Bull. Lond. Math. Soc. 45:5 (2013), 1100-1106. MR 3105002 Zbl 1284.46053

[Blecher 2015] D. P. Blecher, "Generalization of $C^{*}$-algebra methods via real positivity for operator and Banach algebras", preprint, 2015. To appear in Operator algebras and their applications: A tribute to Richard V. Kadison, edited by R. S. Doran and E. Park, Contemporary Mathematics 671, American Mathematical Society. arXiv 1508.03059

[Blecher and Labuschagne 2003] D. P. Blecher and L. E. Labuschagne, "Logmodularity and isometries of operator algebras", Trans. Amer. Math. Soc. 355:4 (2003), 1621-1646. MR 1946408 Zbl 1026.46046

[Blecher and Le Merdy 2004] D. P. Blecher and C. Le Merdy, Operator algebras and their modules: an operator space approach, London Mathematical Society Monographs. New Series 30, Oxford University Press, 2004. MR 2111973 Zbl 1061.47002

[Blecher and Magajna 2005a] D. P. Blecher and B. Magajna, "Duality and operator algebras: automatic weak* continuity and applications", J. Funct. Anal. 224:2 (2005), 386-407. MR 2146046

[Blecher and Magajna 2005b] D. P. Blecher and B. Magajna, "Duality and operator algebras, II: Operator algebras as Banach algebras”, J. Funct. Anal. 226:2 (2005), 485-493. MR 2160105 Zbl 1083.46033

[Blecher and Neal 2012a] D. P. Blecher and M. Neal, "Open projections in operator algebras, I: Comparison theory”, Studia Math. 208:2 (2012), 117-150. MR 2910983 Zbl 1259.46045

[Blecher and Neal 2012b] D. P. Blecher and M. Neal, "Open projections in operator algebras, II: Compact projections”, Studia Math. 209:3 (2012), 203-224. MR 2944468 Zbl 1259.46046

[Blecher and Ozawa 2015] D. P. Blecher and N. Ozawa, "Real positivity and approximate identities in Banach algebras", Pacific J. Math. 277:1 (2015), 1-59. MR 3393680 Zbl 06479248

[Blecher and Read 2011] D. P. Blecher and C. J. Read, "Operator algebras with contractive approximate identities", J. Funct. Anal. 261:1 (2011), 188-217. MR 2785898 Zbl 1235.47087

[Blecher and Read 2013] D. P. Blecher and C. J. Read, "Operator algebras with contractive approximate identities, II”, J. Funct. Anal. 264:4 (2013), 1049-1067. MR 3004957 Zbl 1270.47067

[Blecher and Read 2014] D. P. Blecher and C. J. Read, "Order theory and interpolation in operator algebras”, Studia Math. 225:1 (2014), 61-95. MR 3299396 Zbl 06390240

[Blecher et al. 2008] D. P. Blecher, D. M. Hay, and M. Neal, "Hereditary subalgebras of operator algebras”, J. Oper. Theory 59:2 (2008), 333-357. MR 2411049 Zbl 1164.46018

[Blecher et al. $\geq 2016]$ D. P. Blecher, M. Neal, and Z. Wang, "Jordan operator algebras and their contractive projections", in preparation (tentative title).

[Cabrera García and Rodríguez Palacios 2014] M. Cabrera García and Á. Rodríguez Palacios, Non-associative normed algebras, 1: The Vidav-Palmer and Gelfand-Naimark theorems, Encyclopedia of Mathematics and its Applications 154, Cambridge University Press, 2014. MR 3242640 Zbl 1322.46003

[Choi and Effros 1976] M.-D. Choi and E. G. Effros, "The completely positive lifting problem for $C^{*}$-algebras", Ann. of Math. (2) 104:3 (1976), 585-609. MR 0417795 Zbl 0361.46067 
[Choi and Effros 1977] M.-D. Choi and E. G. Effros, "Injectivity and operator spaces", J. Funct. Anal. 24:2 (1977), 156-209. MR 0430809 Zbl 0341.46049

[Chu et al. 2004] C.-H. Chu, M. Neal, and B. Russo, "Normal contractive projections preserve type", J. Operator Theory 51:2 (2004), 281-301. MR 2074182 Zbl 1113.46066

[Edwards and Rüttimann 1996] C. M. Edwards and G. T. Rüttimann, "Compact tripotents in bidual JB*-triples”, Math. Proc. Cambridge Philos. Soc. 120:1 (1996), 155-173. MR 1373355 Zbl 0853.46070

[Effros 1963] E. G. Effros, "Order ideals in a $C^{*}$-algebra and its dual”, Duke Math. J. 30 (1963), 391-411. MR 0151864 Zbl 0117.09703

[Effros and Størmer 1979] E. G. Effros and E. Størmer, "Positive projections and Jordan structure in operator algebras”, Math. Scand. 45:1 (1979), 127-138. MR 567438 Zbl 0455.46059

[Friedman and Russo 1982] Y. Friedman and B. Russo, "Contractive projections on $C_{0}(K)$ ", Trans. Amer. Math. Soc. 273:1 (1982), 57-73. MR 664029 Zbl 0534.46037

[Friedman and Russo 1984] Y. Friedman and B. Russo, "Conditional expectation without order", Pacific J. Math. 115:2 (1984), 351-360. MR 765191 Zbl 0563.46039

[Friedman and Russo 1985] Y. Friedman and B. Russo, "Solution of the contractive projection problem”, J. Funct. Anal. 60:1 (1985), 56-79. MR 780104 Zbl 0558.46035

[Godefroy et al. 1993] G. Godefroy, N. J. Kalton, and P. D. Saphar, "Unconditional ideals in Banach spaces", Studia Math. 104:1 (1993), 13-59. MR 1208038 Zbl 0814.46012

[Hamana 1999] M. Hamana, “Triple envelopes and Šilov boundaries of operator spaces", Math. J. Toyama Univ. 22 (1999), 77-93. MR 1744498 Zbl 0948.46045

[Harris 1981] L. A. Harris, "A generalization of $C^{*}$-algebras”, Proc. London Math. Soc. (3) 42:2 (1981), 331-361. MR 607306 Zbl 0476.46054

[Isidro and Rodríguez-Palacios 1995] J. M. Isidro and Á. Rodríguez-Palacios, "Isometries of JBalgebras”, Manuscripta Math. 86:3 (1995), 337-348. MR 1323796 Zbl 0834.17048

[Kadison 1951] R. V. Kadison, "Isometries of operator algebras", Ann. of Math. (2) 54 (1951), 325-338. MR 0043392 Zbl 0045.06201

[Kadison 1952] R. V. Kadison, "A generalized Schwarz inequality and algebraic invariants for operator algebras”, Ann. of Math. (2) 56 (1952), 494-503. MR 0051442 Zbl 0047.35703

[Lau and Loy 2008] A. T.-M. Lau and R. J. Loy, "Contractive projections on Banach algebras", J. Funct. Anal. 254:10 (2008), 2513-2533. MR 2406685 Zbl 1149.46040

[Neal 2000] M. Neal, "Inner ideals and facial structure of the quasi-state space of a JB-algebra", $J$. Funct. Anal. 173:2 (2000), 284-307. MR 1760616 Zbl 0959.46039

[Neal and Russo 2000] M. Neal and B. Russo, "Contractive projections and operator spaces", C. R. Acad. Sci. Paris Sér. I Math. 331:11 (2000), 873-878. MR 1806425 Zbl 0973.47052

[Neal and Russo 2003a] M. Neal and B. Russo, "Contractive projections and operator spaces", Trans. Amer. Math. Soc. 355:6 (2003), 2223-2262. MR 1973989 Zbl 1088.46030

[Neal and Russo 2003b] M. Neal and B. Russo, "Operator space characterizations of $C^{*}$-algebras and ternary rings", Pacific J. Math. 209:2 (2003), 339-364. MR 1978376 Zbl 1064.46060

[Neal and Russo 2006] M. Neal and B. Russo, "Representation of contractively complemented Hilbertian operator spaces on the Fock space", Proc. Amer. Math. Soc. 134:2 (2006), 475-485. MR 2176016 Zbl 1089.46034

[Neal and Russo 2011] M. Neal and B. Russo, "Existence of contractive projections on preduals of JBW*-triples”, Israel J. Math. 182 (2011), 293-331. MR 2783974 Zbl 1232.46063 
[Neal et al. 2006] M. Neal, É. Ricard, and B. Russo, "Classification of contractively complemented Hilbertian operator spaces”, J. Funct. Anal. 237:2 (2006), 589-616. MR 2230351 Zbl 1106.46043

[Paulsen 2002] V. I. Paulsen, Completely bounded maps and operator algebras, Cambridge Studies in Advanced Mathematics 78, Cambridge University Press, 2002. MR 1976867 Zbl 1029.47003

[Pedersen 1979] G. K. Pedersen, $C^{*}$-algebras and their automorphism groups, London Mathematical Society Monographs 14, Academic Press, London, 1979. MR 548006 Zbl 0416.46043

[Robertson and Youngson 1982] A. G. Robertson and M. A. Youngson, "Positive projections with contractive complements on Jordan algebras", J. London Math. Soc. (2) 25:2 (1982), 365-374. MR 653394 Zbl 0481.46032

[Russo 1994] B. Russo, "Structure of JB*-triples", pp. 209-280 in Jordan algebras (Oberwolfach, 1992), edited by W. Kaup and K. McCrimmon, de Gruyter, Berlin, 1994. MR 1293321 Zbl 0805.46072

[Størmer 1982] E. Størmer, "Positive projections with contractive complements on $C^{*}$-algebras", $J$. London Math. Soc. (2) 26:1 (1982), 132-142. MR 667251 Zbl 0501.46048

[Youngson 1983] M. A. Youngson, "Completely contractive projections on $C^{*}$-algebras", Quart. J. Math. Oxford Ser. (2) 34:136 (1983), 507-511. MR 723287 Zbl 0542.46029

Received August 6, 2015. Revised January 11, 2016.

DAVID P. BLECHER

DEPARTMENT OF MATHEMATICS

UNIVERSITY OF HOUSTON

Houston, TX 77204-3008

UNITED STATES

dblecher@math.uh.edu

MATTHEW NeAL

DEPARTMENT OF MATHEMATICS

DENISON UNIVERSITY

GRANVILLE, OH 43023

UNITED STATES

nealm@denison.edu 


\title{
PACIFIC JOURNAL OF MATHEMATICS
}

Founded in 1951 by E. F. Beckenbach (1906-1982) and F. Wolf (1904-1989)

$$
\text { msp.org/pjm }
$$

\section{EDITORS}

\author{
Don Blasius (Managing Editor) \\ Department of Mathematics \\ University of California \\ Los Angeles, CA 90095-1555 \\ blasius@math.ucla.edu
}

\author{
Paul Balmer \\ Department of Mathematics \\ University of California \\ Los Angeles, CA 90095-1555 \\ balmer@math.ucla.edu \\ Robert Finn \\ Department of Mathematics \\ Stanford University \\ Stanford, CA 94305-2125 \\ finn@math.stanford.edu \\ Sorin Popa \\ Department of Mathematics \\ University of California \\ Los Angeles, CA 90095-1555 \\ popa@math.ucla.edu
}

\author{
Vyjayanthi Chari \\ Department of Mathematics \\ University of California \\ Riverside, CA 92521-0135 \\ chari@math.ucr.edu \\ Kefeng Liu \\ Department of Mathematics \\ University of California \\ Los Angeles, CA 90095-1555 \\ liu@math.ucla.edu \\ Igor Pak \\ Department of Mathematics \\ University of California \\ Los Angeles, CA 90095-1555 \\ pak.pjm@gmail.com \\ Paul Yang \\ Department of Mathematics \\ Princeton University \\ Princeton NJ 08544-1000 \\ yang@math.princeton.edu
}

\section{PRODUCTION}

Silvio Levy, Scientific Editor, production@msp.org

\section{SUPPORTING INSTITUTIONS}

ACADEMIA SINICA, TAIPEI

CALIFORNIA INST. OF TECHNOLOGY

STANFORD UNIVERSITY

UNIV. OF BRITISH COLUMBIA

UNIV. OF CALIFORNIA, BERKELEY

UNIV. OF CALIFORNIA, DAVIS

UNIV. OF CALIFORNIA, LOS ANGELES

UNIV. OF CALIFORNIA, RIVERSIDE

UNIV. OF CALIFORNIA, SAN DIEGO

UNIV. OF CALIF., SANTA BARBARA
KEIO UNIVERSITY

MATH. SCIENCES RESEARCH INSTITUTE

NEW MEXICO STATE UNIV.

OREGON STATE UNIV.
Daryl Cooper

Department of Mathematics

University of California

Santa Barbara, CA 93106-3080 cooper@math.ucsb.edu

Jiang-Hua Lu

Department of Mathematics

The University of Hong Kong

Pokfulam Rd., Hong Kong

jhlu@maths.hku.hk

$$
\text { Jie Qing }
$$

Department of Mathematics

University of California

Santa Cruz, CA 95064

qing@ cats.ucsc.edu

\author{
UNIV. OF CALIF., SANTA CRUZ \\ UNIV. OF MONTANA \\ UNIV. OF OREGON \\ UNIV. OF SOUTHERN CALIFORNIA \\ UNIV. OF UTAH \\ UNIV. OF WASHINGTON \\ WASHINGTON STATE UNIVERSITY
}

These supporting institutions contribute to the cost of publication of this Journal, but they are not owners or publishers and have no responsibility for its contents or policies.

See inside back cover or msp.org/pjm for submission instructions.

The subscription price for 2016 is US $\$ 440 /$ year for the electronic version, and \$600/year for print and electronic.

Subscriptions, requests for back issues and changes of subscriber address should be sent to Pacific Journal of Mathematics, P.O. Box 4163, Berkeley, CA 94704-0163, U.S.A. The Pacific Journal of Mathematics is indexed by Mathematical Reviews, Zentralblatt MATH, PASCAL CNRS Index, Referativnyi Zhurnal, Current Mathematical Publications and Web of Knowledge (Science Citation Index).

The Pacific Journal of Mathematics (ISSN 0030-8730) at the University of California, c/o Department of Mathematics, 798 Evans Hall \#3840, Berkeley, CA 94720-3840, is published twelve times a year. Periodical rate postage paid at Berkeley, CA 94704, and additional mailing offices. POSTMASTER: send address changes to Pacific Journal of Mathematics, P.O. Box 4163, Berkeley, CA 94704-0163.

PJM peer review and production are managed by EditFLOW ${ }^{\circledR}$ from Mathematical Sciences Publishers.

PUBLISHED BY

\section{I. mathematical sciences publishers}

nonprofit scientific publishing

http://msp.org/

(C) 2016 Mathematical Sciences Publishers 


\section{PACIFIC JOURNAL OF MATHEMATICS}

Volume $283 \quad$ No. $2 \quad$ August 2016

The fundamental theorem of tropical differential algebraic geometry

FUENSANTA AROCA, CRISTHIAN GARAY and ZEINAB TOGHANI

A simple solution to the word problem for virtual braid groups

271

PaOlo Bellingeri, Bruno A. Cisneros de la CruZ and Luis

PARIS

Completely contractive projections on operator algebras

DAVID P. BLECHER and MATTHEW NEAL

Invariants of some compactified Picard modular surfaces and applications

AMIR DŽAMBIĆ

Radial limits of bounded nonparametric prescribed mean curvature surfaces

MOZHGAN (NORA) ENTEKHABI and KIRK E. LANCASTER

A remark on the Noetherian property of power series rings

BYUNG GYUN KANG and PHAN THANH TOAN

Curves with prescribed intersection with boundary divisors in moduli spaces of curves

\section{XIAO-LEI LIU}

Virtual rational Betti numbers of nilpotent-by-abelian groups

BEHROOZ MiRZAII and FATEMEH Y. MOKARI

A planar Sobolev extension theorem for piecewise linear homeomorphisms 405

EMANUELA RADICI

A combinatorial approach to Voiculescu's bi-free partial transforms

PAUL SKOUFRANIS

Vector bundle valued differential forms on $\mathbb{N} Q$-manifolds

LUCA VitAGLiANO

Discriminants and the monoid of quadratic rings 